\title{
On the existence of almost greedy bases in Banach spaces
}

\author{
by
S. J. Dilworth (Columbia, SC), N. J. Kalton (Columbia, MO), and Denka Kutzarova (Sofia and Urbana, IL)

\begin{abstract}
We consider several greedy conditions for bases in Banach spaces that arise naturally in the study of the Thresholding Greedy Algorithm (TGA). In particular, we continue the study of almost greedy bases begun in [3]. We show that almost greedy bases are essentially optimal for $n$-term approximation when the TGA is modified to include a Chebyshev approximation. We prove that if a Banach space $X$ has a basis and contains a complemented subspace with a symmetric basis and finite cotype then $X$ has an almost greedy basis. We show that $c_{0}$ is the only $\mathcal{L}_{\infty}$ space to have a quasi-greedy basis. The Banach spaces which contain almost greedy basic sequences are characterized.
\end{abstract}

1. Introduction. Let $X$ be a real Banach space with a semi-normalized basis $\left(e_{n}\right)$. An algorithm for $n$-term approximation produces a sequence of maps $F_{n}: X \rightarrow X$ such that, for each $x \in X, F_{n}(x)$ is a linear combination of at most $n$ of the basis elements $\left(e_{j}\right)$. The most natural algorithm is the linear algorithm $\left(S_{n}\right)_{n=1}^{\infty}$ given by the partial sum operators.

Recently, Konyagin and Temlyakov [12] introduced the Thresholding Greedy Algorithm (TGA) $\left(G_{n}\right)_{n=1}^{\infty}$, where $G_{n}(x)$ is obtained by taking the largest $n$ coefficients (precise definitions are given in Section 2). The TGA provides a theoretical model for the thresholding procedure that is used in image compression and other applications.

They defined the basis $\left(e_{n}\right)$ to be greedy if the TGA is optimal in the sense that $G_{n}(x)$ is essentially the best $n$-term approximation to $x$ using the basis vectors, i.e. if there exists a constant $C$ such that for all $x \in X$ and $n \in \mathbb{N}$, we have

$$
\left\|x-G_{n}(x)\right\| \leq C \inf \left\{\left\|x-\sum_{j \in A} \alpha_{j} e_{j}\right\|:|A|=n, \alpha_{j} \in \mathbb{R}\right\} .
$$

2000 Mathematics Subject Classification: Primary 46B15; Secondary 41A65, 46B20.

The research of the first author was supported by a summer research grant from the University of South Carolina in July 2002 while on sabbatical as a Visiting Scholar at The University of Texas at Austin for the academic year 2001-2002. The research of the second author was supported by NSF grant DMS-9870027. 
They then showed (see Theorem 2.3 below) that greedy bases can be simply characterized as unconditional bases with the additional property of being democratic, i.e. for some $\Delta>0$, we have

$$
\left\|\sum_{j \in A} e_{j}\right\| \leq \Delta\left\|\sum_{j \in B} e_{j}\right\| \quad \text { whenever }|A| \leq|B| .
$$

They also defined a basis to be quasi-greedy if there exists a constant $C$ such that $\left\|G_{m}(x)\right\| \leq C\|x\|$ for all $x \in X$ and $n \in \mathbb{N}$. Subsequently, Wojtaszczyk [23] proved that these are precisely the bases for which the TGA merely converges, i.e. $\lim _{n \rightarrow \infty} G_{n}(x)=x$ for $x \in X$.

The class of almost greedy bases was introduced in [3]. Let us denote the biorthogonal sequence by $\left(e_{n}^{*}\right)$. Then $\left(e_{n}\right)$ is almost greedy if there exists a constant $C$ such that for all $x \in X$ and $n \in \mathbb{N}$, we have

$$
\left\|x-G_{n}(x)\right\| \leq C \inf \left\{\left\|x-\sum_{j \in A} e_{j}^{*}(x) e_{j}\right\|:|A|=n\right\} .
$$

Comparison with (1.1) shows that this is formally a weaker condition: in (1.1) the infimum is taken over all possible $n$-term approximations, while in (1.2) only projections of $x$ onto the basis vectors are considered. It was proved in [3] (see Theorem 2.5 below) that $\left(e_{n}\right)$ is almost greedy if and only if $\left(e_{n}\right)$ is quasi-greedy and democratic.

In this paper we continue the study of almost greedy bases and related greedy conditions for bases. In Section 3 we consider a natural modification of the TGA which improves the rate of convergence. Let $G_{n}^{C}(x)$ be an $n$-term Chebyshev approximation to $x$ using the basis vectors given by the TGA, i.e., those with the largest $n$ coefficients. We show that if $\left(e_{n}\right)$ is almost greedy, then $G_{n}^{C}(x)$ is essentially the best $n$-term approximation in the sense described above. For Banach spaces with finite cotype, we also show that the latter property characterizes almost greedy bases.

In Section 4 we consider the thresholding operators:

$$
\mathcal{G}_{a}(x)=\sum_{\left|e_{i}^{*}(x)\right| \geq a} e_{i}^{*}(x) e_{i} \quad(a>0, x \in X) .
$$

There are natural boundedness conditions to impose on these operators and a corresponding class of thresholding-bounded bases which satisfy these conditions. We show that this class coincides with the class of nearly unconditional bases introduced by Elton [5] and that it strictly contains the class of quasi-greedy bases.

In Section 5 we prove existence results for almost greedy basic sequences. In particular, we give necessary and sufficient conditions for a semi-normalized weakly null sequence to have an almost greedy subsequence and we characterize the Banach spaces which contain almost greedy basic sequences. 
The rest of the paper concerns the existence and nonexistence of quasigreedy and almost greedy bases (as opposed to basic sequences). The results contained in Sections 6-7 extend a theorem of Wojtaszczyk [23]. We prove that if $X$ has a basis and contains a complemented subspace with a symmetric basis and finite cotype, then $X$ has an almost greedy basis. More generally, we show that if $X$ has a basis and contains a complemented "good" (loosely, "far from $c_{0}$ ") unconditional basic sequence, then $X$ has a quasi-greedy basis. The fact that there is no corresponding result for $c_{0}$ is explained by the last section of the paper.

Section 8 contains the nonexistence results. We prove that $c_{0}$ is the only $\mathcal{L}_{\infty}$ space to have a quasi-greedy basis. Thus, $C[0,1]$ and (by similar reasoning) the disc algebra do not have quasi-greedy bases. Lastly, we deduce from the Lindenstrauss-Zippin theorem [14] that $c_{0}$ is the only infinitedimensional Banach space up to isomorphism to have a unique quasi-greedy basis up to equivalence.

Standard Banach space notation and terminology are used throughout (see [13]). For clarity, however, we record the notation that is used most heavily. We write $X \sim Y$ if $X$ and $Y$ are linearly isomorphic Banach spaces. We say that $X$ and $Y$ are $\lambda$-isomorphic if there exists an isomorphism $T$ : $X \rightarrow Y$ with $\|T\|\left\|T^{-1}\right\| \leq \lambda$. A subspace $Z$ of $X$ is said to be complemented if $Z$ is the range of a continuous linear projection on $X$.

Let $\left(x_{n}\right)$ be a sequence in $X$. We say that $\left(x_{n}\right)$ is semi-normalized (resp. normalized) if there exists $C>0$ such that $1 / C \leq\left\|x_{n}\right\| \leq C$ (resp. $\left\|x_{n}\right\|=$ $1)$ for all $n \geq 1$. The closed linear span of $\left(x_{n}\right)$ is denoted by $\left[x_{n}\right]$. We say that a sequence $\left(x_{n}\right)$ of nonzero vectors is basic if there exists a positive constant $K$ such that

$$
\left\|\sum_{i=1}^{m} a_{i} x_{i}\right\| \leq K\left\|\sum_{i=1}^{n} a_{i} x_{i}\right\|
$$

for all scalars $\left(a_{i}\right)$ and all $1 \leq m \leq n \in \mathbb{N} ;\left(x_{n}\right)$ is monotone if we can take $K=1 ;\left(x_{n}\right)$ is $\lambda$-unconditional if

$$
\left\|\sum_{i=1}^{\infty} \varepsilon_{i} a_{i} x_{i}\right\| \leq \lambda\left\|\sum_{i=1}^{\infty} a_{i} x_{i}\right\|
$$

for all scalars $\left(a_{i}\right)$ and all choices of signs $\varepsilon_{i}= \pm 1$. We say that $\left(x_{n}\right)$ is $\lambda$-symmetric if

$$
\left\|\sum_{i=1}^{\infty} a_{\sigma(i)} x_{i}\right\| \leq \lambda\left\|\sum_{i=1}^{\infty} a_{i} x_{i}\right\|
$$

for all permutations $\sigma$ of $\mathbb{N}$. A basis for $X$ is a sequence $\left(e_{n}\right)$ of vectors such that every $x \in X$ has a unique expansion as a norm-convergent series 


$$
x=\sum_{i=1}^{\infty} e_{i}^{*}(x) e_{i},
$$

where $\left(e_{i}^{*}\right)$ is the sequence of biorthogonal functionals in the dual space $X^{*}$ defined by $e_{i}^{*}\left(e_{j}\right)=\delta_{i, j}$. The usual norms of the sequence spaces $\ell_{p}$ and $\ell_{\infty}$ are denoted by $\|\cdot\|_{p}$ and $\|\cdot\|_{\infty}$. The sequence space $c_{00}$ consists of all sequences with only finitely many nonzero terms. For a sequence $\left(X_{n},\|\cdot\|_{n}\right)$ of Banach spaces, the direct sum $\left(\sum_{n=1}^{\infty} \oplus X_{n}\right)_{p}$ is the space of all sequences $\left(x_{n}\right)\left(x_{n} \in X_{n}\right)$ equipped with the norm

$$
\left\|\left(x_{n}\right)\right\|=\left(\sum_{n=1}^{\infty}\left\|x_{n}\right\|_{n}^{p}\right)^{1 / p}
$$

More specialized notions from Banach space theory will be introduced as needed.

Finally, it is worth emphasizing that we consider only real Banach spaces in this paper.

2. Preliminaries. Let $\left(e_{n}\right)$ be a semi-normalized basis of a Banach space $X$, and let $\left(e_{n}^{*}\right)$ be the biorthogonal sequence in $X^{*}$. For $x \in X$, we define the greedy ordering for $x$ as the map $\varrho: \mathbb{N} \rightarrow \mathbb{N}$ such that $\varrho(\mathbb{N}) \supset\{j$ : $\left.e_{j}^{*}(x) \neq 0\right\}$ and such that if $j<k$ then

$$
\left|e_{\varrho(j)}^{*}(x)\right|>\left|e_{\varrho(k)}^{*}(x)\right| \quad \text { or } \quad e_{\varrho(j)}^{*}(x)|=| e_{\varrho(k)}^{*}(x) \mid \text { and } \varrho(j)<\varrho(k) .
$$

The $m$ th greedy approximation is given by

$$
G_{m}(x)=\sum_{j=1}^{m} e_{\varrho(j)}^{*}(x) e_{\varrho(j)} .
$$

The basis $\left(e_{n}\right)$ is called quasi-greedy if $G_{m}(x) \rightarrow x$ for all $x \in X$. This is equivalent (see [23]) to the condition that for some constant $K$ we have

$$
\sup _{m}\left\|G_{m}(x)\right\| \leq K\|x\| \quad(x \in X) .
$$

We define the quasi-greedy constant $K$ to be the least such constant.

The following two lemmas are essentially due to Wojtaszczyk [23]. For proofs we refer to [3] or to Section 4 below for slightly more general "localized" versions of the same results. Note that (2.4) says that a quasi-greedy basis is unconditional for constant coefficients.

LEMMA 2.1. Suppose that $\left(e_{n}\right)$ has quasi-greedy constant $K$ and that $A$ is a finite subset of $\mathbb{N}$. Then, for every choice of signs $\varepsilon_{j}= \pm 1$, we have

$$
\frac{1}{2 K}\left\|\sum_{j \in A} e_{j}\right\| \leq\left\|\sum_{j \in A} \varepsilon_{j} e_{j}\right\| \leq 2 K\left\|\sum_{j \in A} e_{j}\right\|,
$$


and hence for any real numbers $\left(a_{j}\right)_{j \in A}$,

$$
\left\|\sum_{j \in A} a_{j} e_{j}\right\| \leq 2 K \max _{j \in A}\left|a_{j}\right|\left\|\sum_{j \in A} e_{j}\right\| .
$$

Lemma 2.2. Suppose that $\left(e_{n}\right)$ has quasi-greedy constant $K$ and that $x \in X$ has greedy ordering $\varrho$. Then

$$
\left|e_{\varrho(m)}^{*}(x)\right||| \sum_{j=1}^{m}\left(\operatorname{sgn} e_{\varrho(j)}^{*}(x)\right) e_{\varrho(j)}\|\leq 2 K\| x \| .
$$

Hence if $A \subset \mathbb{N}$ is finite and $\left(a_{j}\right)_{j \in A}$ are any real numbers,

$$
\min _{j \in A}\left|a_{j}\right|\left\|\sum_{j \in A}\left(\operatorname{sgn} a_{j}\right) e_{j}\right\| \leq(1+K)\left\|\sum_{j \in A} a_{j} e_{j}\right\| .
$$

For a Schauder basis $\left(e_{n}\right)$ we define

$$
\sigma_{m}(x):=\inf \left\{\left\|x-\sum_{j \in A} \alpha_{j} e_{j}\right\|:|A|=m, \alpha_{j} \in \mathbb{R}\right\} .
$$

A basis $\left(e_{n}\right)$ is called greedy [12] if there exists a constant $C$ such that for any $x \in X$ and $m \in \mathbb{N}$ we have

$$
\left\|x-G_{m}(x)\right\| \leq C \sigma_{m}(x) .
$$

A basis $\left(e_{n}\right)$ is called democratic [12] if there is a constant $\Delta$ such that

$$
\left\|\sum_{k \in A} e_{k}\right\| \leq \Delta\left\|\sum_{k \in B} e_{k}\right\| \quad \text { if }|A| \leq|B| .
$$

Note that a democratic basis is automatically semi-normalized.

The following characterization of greedy bases was proved in [12].

Theorem 2.3. A basis $\left(e_{n}\right)$ is greedy if and only if it is unconditional and democratic.

For a semi-normalized basis $\left(e_{n}\right)$ we define the fundamental function $\varphi(n)$ by

$$
\varphi(n)=\sup _{|A| \leq n}\left\|\sum_{k \in A} e_{k}\right\|
$$

Note that $\varphi$ is subadditive (i.e. $\varphi(m+n) \leq \varphi(m)+\varphi(n))$ and increasing. It may also be seen that $\varphi(n) / n$ is decreasing since for any set $A$ with $|A|=n$, we have

$$
\sum_{k \in A} e_{k}=\frac{1}{n-1} \sum_{k \in A} \sum_{j \neq k} e_{j} .
$$

It follows that for any finite $A \subset \mathbb{N}$ and any real scalars $\left(a_{j}\right)_{j \in A}$ we have

$$
\left\|\sum_{j \in A} a_{j} e_{j}\right\| \leq 2 \varphi(|A|) \max _{j \in A}\left|a_{j}\right| .
$$


It is clear that if $\left(e_{k}\right)$ is democratic with constant $\Delta$ in $(2.9)$ then

$$
\Delta^{-1} \varphi(|A|) \leq\left\|\sum_{k \in A} e_{k}\right\| \leq \varphi(|A|) \quad(|A|<\infty) .
$$

Combining (2.4), (2.6), and (2.9) yields the following estimate (cf. [6]).

LEMMA 2.4. Let $\left(e_{n}\right)$ be a democratic quasi-greedy basis with quasigreedy constant $K$ and democratic constant $\Delta$. Then for $x \in X$ with greedy ordering $\varrho$, we have

$$
\left|e_{\varrho(m)}^{*}(x)\right| \leq \frac{4 K^{2} \Delta}{\varphi(m)}\|x\| .
$$

A slightly weaker form of greediness was introduced in [3]. For a basis $\left(e_{n}\right)$, let

$$
\widetilde{\sigma}_{m}(x):=\inf \left\{\left\|x-\sum_{k \in A} e_{k}^{*}(x) e_{k}\right\|:|A| \leq m\right\} .
$$

Note that

$$
\sigma_{m}(x) \leq \widetilde{\sigma}_{m}(x) \leq\left\|x-S_{m}(x)\right\| \rightarrow 0 \quad \text { as } m \rightarrow \infty .
$$

We say that a basis $\left(e_{n}\right)$ is almost greedy if there exists a constant $C$ such that for all $x \in X$ we have

$$
\left\|x-G_{m}(x)\right\| \leq C \widetilde{\sigma}_{m}(x) .
$$

The following characterization of almost greedy bases was proved in [3].

Theorem 2.5. Suppose that $\left(e_{n}\right)$ is a basis of a Banach space. The following are equivalent:

(1) $\left(e_{n}\right)$ is almost greedy.

(2) $\left(e_{n}\right)$ is quasi-greedy and democratic.

(3) For some (respectively, every) $\lambda>1$ there exists a constant $C_{\lambda}$ such that

$$
\left\|x-G_{[\lambda m]} x\right\| \leq C_{\lambda} \sigma_{m}(x)
$$

Most of the democratic bases which we consider in this paper actually satisfy a stronger property. Following [12], we say that a basis $\left(e_{n}\right)$ is $s u$ perdemocratic if there exists a constant $C$ such that for all finite $A, B \subseteq \mathbb{N}$, and for all choices of signs $\left(\varepsilon_{i}\right)_{i \in A}$ and $\left(\eta_{i}\right)_{i \in B}$, we have

$$
\frac{1}{C}\left\|\sum_{i \in B} \eta_{i} e_{i}\right\| \leq\left\|\sum_{i \in A} \varepsilon_{i} e_{i}\right\| \leq C\left\|\sum_{i \in B} \eta_{i} e_{i}\right\| .
$$

It is easy to see that a basis is superdemocratic if and only if it is democratic and unconditional for constant coefficients. By (2.4), every almost greedy basis is superdemocratic. An example of a basis that is superdemocratic but not quasi-greedy is given below (Example 4.8). 
3. Semi-greedy bases. In this section we consider an obvious enhancement of the TGA which improves the rate of convergence. Suppose that $x \in$ $X$ and let $\varrho$ be the greedy ordering for $x$. Let $G_{n}^{\mathrm{C}}(x) \in \operatorname{span}\left\{e_{\varrho(i)}: 1 \leq i \leq n\right\}$ be a Chebyshev approximation to $x$. Thus,

$$
\left\|x-G_{n}^{\mathrm{C}}(x)\right\|=\min \left\{\left\|x-\sum_{i=1}^{n} a_{i} e_{\varrho(i)}\right\|:\left(a_{i}\right)_{i=1}^{n} \in \mathbb{R}^{n}\right\} .
$$

It is natural to make the following definition. Let $\left(e_{n}\right)$ be a semi-normalized basis for $X$. We say that $\left(e_{n}\right)$ is semi-greedy if there exists a constant $C$ such that for all $n \geq 1$ and for all $x \in X$ we have

$$
\left\|x-G_{n}^{\mathrm{C}}(x)\right\| \leq C \sigma_{n}(x) .
$$

We prove that an almost greedy basis is always semi-greedy and that the converse holds for Banach spaces with finite cotype. The proof of the former result uses the fact that the norm in a space with a quasi-greedy basis behaves well under "truncation of coefficients".

Fix $M>0$. Define the "truncation function" $f_{M}: \mathbb{R} \rightarrow[-M, M]$ thus:

$$
f_{M}(x)= \begin{cases}M & \text { for } x>M \\ x & \text { for } x \in[-M, M] \\ -M & \text { for } x<-M\end{cases}
$$

Proposition 3.1. Suppose that $\left(e_{n}\right)$ is quasi-greedy with quasi-greedy constant $K$. Then, for every $M>0$ and for all real scalars $\left(a_{i}\right)$, we have

$$
\left\|\sum_{i=1}^{\infty} f_{M}\left(a_{i}\right) e_{i}\right\| \leq(1+3 K)\left\|\sum_{i=1}^{\infty} a_{i} e_{i}\right\| .
$$

Proof. Let $x=\sum_{i=1}^{\infty} a_{i} e_{i}$ and let $\varrho$ be the greedy ordering for $x$. If $M>\max \left|a_{i}\right|$ there is nothing to prove. So suppose that there exists $N$ such that

$$
\left|a_{\varrho(N+1)}\right|<M \leq\left|a_{\varrho(N)}\right| .
$$

Then

$$
\begin{aligned}
\left\|\sum_{i=1}^{N} f_{M}\left(a_{\varrho(i)}\right) e_{\varrho(i)}\right\| & =M\left\|\sum_{i=1}^{N}\left(\operatorname{sgn} a_{\varrho(i)}\right) e_{\varrho(i)}\right\| \\
& \leq\left|a_{\varrho(N)}\right|\left\|\sum_{i=1}^{N}\left(\operatorname{sgn} a_{\varrho(i)}\right) e_{\varrho(i)}\right\| \leq 2 K\|x\|
\end{aligned}
$$

by Lemma 2.2. Moreover,

$$
\left\|\sum_{i=N+1}^{\infty} f_{M}\left(a_{\varrho(i)}\right) e_{\varrho(i)}\right\|=\left\|\sum_{i=N+1}^{\infty} a_{\varrho(i)} e_{\varrho(i)}\right\|=\left\|x-G_{N}(x)\right\| \leq(1+K)\|x\| .
$$


Combining, we get

$$
\begin{aligned}
\left\|\sum_{i=1}^{\infty} f_{M}\left(a_{i}\right) e_{i}\right\| & \leq\left\|\sum_{i=1}^{N} f_{M}\left(a_{\varrho(i)}\right) e_{\varrho(i)}\right\|+\left\|\sum_{i=N+1}^{\infty} f_{M}\left(a_{\varrho(i)}\right) e_{\varrho(i)}\right\| \\
& \leq(1+3 K)\|x\| . \text { - }
\end{aligned}
$$

TheOrem 3.2. Every almost greedy basis is semi-greedy.

Proof. By Theorem 2.5, $\left(e_{n}\right)$ is quasi-greedy and democratic. Let $K$ be the quasi-greedy and $\Delta$ the democratic constant of $\left(e_{n}\right)$. Fix $n \geq 1$ and $x=$ $\sum_{i=1}^{\infty} a_{i} e_{i}$ in $X$. Let $\varrho$ be the greedy ordering for $x$. Let $A=\{\varrho(1), \ldots, \varrho(n)\}$ and let $z:=\sum_{i \in B} b_{i} e_{i}$ be a good $n$-term approximation to $x$, with $|B|=n$ and

$$
\|x-z\| \leq 2 \sigma_{n}(x) .
$$

If $A=B$ there is nothing to prove. So we may assume that $A \backslash B$ is nonempty. Let $k=|A \backslash B|$, so $1 \leq k \leq n$, and let $M=\left|a_{\varrho(n)}\right|$. Then by (2.12),

$$
M \phi(k) \leq 4 K^{2} \Delta\|x-z\|,
$$

since $\left|e_{i}^{*}(x-z)\right| \geq M$ for all $i \in A \backslash B$. Let

$$
x-z:=\sum_{i=1}^{\infty} y_{i} e_{i}
$$

By Proposition 3.1, we have

$$
\left\|\sum_{i=1}^{\infty} f_{M}\left(y_{i}\right) e_{i}\right\| \leq(1+3 K)\|x-z\| .
$$

Note that

$$
w:=\sum_{i \in A} f_{M}\left(y_{i}\right) e_{i}+\sum_{i \in \mathbb{N} \backslash A} a_{i} e_{i}=\sum_{i=1}^{\infty} f_{M}\left(y_{i}\right) e_{i}+\sum_{i \in B \backslash A}\left(a_{i}-f_{M}\left(y_{i}\right)\right) e_{i} .
$$

Thus,

$$
\begin{aligned}
\|w\| & \leq\left\|\sum_{i=1}^{\infty} f_{M}\left(y_{i}\right) e_{i}\right\|+\left\|\sum_{i \in B \backslash A}\left(a_{i}-f_{M}\left(y_{i}\right)\right) e_{i}\right\| \\
& \leq(1+3 K)\|x-z\|+4 M \phi(k) \quad \text { (by }(3.15) \text { and }(2.10) \\
& \left.\quad \text { since }\left|a_{i}-f_{M}\left(y_{i}\right)\right| \leq 2 M \text { for } i \in B \backslash A\right) \\
& \leq 2(1+3 K) \sigma_{n}(x)+16 K^{2} \Delta\|x-z\| \quad(\text { by }(3.14)) \\
& \leq\left(2(1+3 K)+32 K^{2} \Delta\right) \sigma_{n}(x) .
\end{aligned}
$$

Since $w=x-\sum_{i \in A}\left(a_{i}-f_{M}\left(y_{i}\right)\right) e_{i}$, we conclude that $\left(e_{n}\right)$ is semi-greedy. 
Next we discuss the converse of Theorem 3.2. It is convenient to introduce the following notation. For finite sets $A, B \subset \mathbb{N}$, we write $A<B$ if $\max \{n$ : $n \in A\}<\min \{n: n \in B\}$.

Proposition 3.3. Every semi-normalized semi-greedy basis $\left(e_{n}\right)$ is superdemocratic.

Proof. Suppose that $|A|=|B|=n$ and let $\left(\varepsilon_{i}\right)$ be any choice of signs. Choose $\varepsilon>0$ and $D \subset \mathbb{N}$, with $A \cup B<D$ and $|D|=n$. Consider

$$
x=\sum_{i \in A} \varepsilon_{i} e_{i}+(1+\varepsilon) \sum_{i \in D} e_{i} .
$$

Since $\left(e_{n}\right)$ is semi-greedy, we have

$$
\left\|\sum_{i \in A} \varepsilon_{i} e_{i}+\sum_{i \in D} c_{i} e_{i}\right\| \leq C \sigma_{n}(x)
$$

for some real scalars $\left(c_{i}\right)$. Hence

$$
\sigma_{n}(x) \leq\left\|\sum_{i \in A} \varepsilon_{i} e_{i}\right\| \leq K\left\|\sum_{i \in A} \varepsilon_{i} e_{i}+\sum_{i \in D} c_{i} e_{i}\right\| \leq C K \sigma_{n}(x),
$$

where $K$ is the basis constant of $\left(e_{n}\right)$. Now consider

$$
y=(1+\varepsilon) \sum_{i \in A} \varepsilon_{i} e_{i}+\sum_{i \in D} e_{i}
$$

A similar argument gives

$$
\sigma_{n}(y) \leq\left\|\sum_{i \in D} e_{i}\right\| \leq C(1+K) \sigma_{n}(y)
$$

Since $\|y-x\| \rightarrow 0$ as $\varepsilon \rightarrow 0$, we obtain

$$
\frac{1}{C K}\left\|\sum_{i \in A} \varepsilon_{i} e_{i}\right\| \leq\left\|\sum_{i \in D} e_{i}\right\| \leq C(K+1)\left\|\sum_{i \in A} \varepsilon_{i} e_{i}\right\| .
$$

The above inequalities also hold with $A$ replaced by $B$. Hence $\left(e_{n}\right)$ is superdemocratic.

Remark 3.4. Let $\left(e_{n}\right)$ be a semi-greedy basis. The proof of Proposition 3.3 actually shows that $\left(e_{n}\right)$ has a fundamental function $(\varphi(n))$ and that there exists a constant $C$ such that

$$
\phi(|A|) \min \left|a_{i}\right| \leq C\left\|\sum_{i \in A} a_{i} e_{i}\right\|
$$

for all finite $A \subset \mathbb{N}$ and all scalars $a_{i}, i \in A$.

A democratic basis $\left(e_{n}\right)$ is said to have the lower regularity property (LRP) if its fundamental function satisfies $C \varphi(m n) \geq m^{\alpha} \varphi(n)$ for all $m, n \in$ $\mathbb{N}$, where $C$ and $0<\alpha \leq 1$ are constants. 
Let us recall that a Banach space $X$ has cotype $q$, where $2 \leq q<\infty$, if there exists a constant $C$ such that

$$
\left(\sum_{j=1}^{n}\left\|x_{j}\right\|^{q}\right)^{1 / q} \leq C\left(\underset{\varepsilon_{j}= \pm 1}{\operatorname{Ave}}\left\|\sum_{j=1}^{n} \varepsilon_{j} x_{j}\right\|^{q}\right)^{1 / q}
$$

for all $x_{1}, \ldots, x_{n} \in X$ and $n \in \mathbb{N}$. The least such constant $C$ is called the cotype $q$ constant $C_{q}(X)$. We say that $X$ has finite cotype if $X$ has cotype $q$ for some $q<\infty$.

Proposition 3.5. Let $\left(e_{n}\right)$ be a superdemocratic basis for a Banach space $X$ which has finite cotype. Then $\left(e_{n}\right)$ has the LRP.

Proof. Suppose that $X$ has cotype $q$. Since $\left(e_{n}\right)$ is superdemocratic there exists a constant $C$ such that for all $m, n \in \mathbb{N}$,

$$
C \varphi(m n) \geq \underset{\varepsilon_{j}= \pm 1}{\operatorname{Ave}}\left\|\sum_{i=0}^{m-1} \varepsilon_{j} \sum_{j=i n+1}^{(i+1) n} e_{i}\right\| \geq\left(1 / C C_{q}\right) m^{1 / q} \varphi(n) .
$$

The last theorem of this section is a partial converse to Theorem 3.2.

Theorem 3.6. Suppose that $\left(e_{n}\right)$ is a semi-greedy basis for a Banach space $X$ which has finite cotype. Then $\left(e_{n}\right)$ is almost greedy.

Proof. We shall not keep track of the constants, so $C$ will denote a constant whose value changes from line to line.

Suppose that $x=\sum_{i \in F} a_{i} e_{i},\|x\|=1$, and $|F|=n$. By Proposition 3.3, it suffices to prove that $\left(e_{n}\right)$ is quasi-greedy, i.e. that $\left\|G_{k}(x)\right\| \leq C$ for $1 \leq k \leq n$. Let $\varrho$ be the greedy ordering for $x$. Since $\left(e_{i}\right)$ is democratic, we have (cf. (2.10))

$$
\left\|x-G_{k}(x)\right\| \leq 2\left|a_{\varrho(k)}\right| \varphi(n-k) .
$$

By Remark 3.4,

$$
\left\|G_{k}(x)\right\| \geq \frac{1}{C}\left|a_{\varrho(k)}\right| \varphi(k) .
$$

Hence

$$
\frac{\left\|x-G_{k}(x)\right\|}{\left\|G_{k}(x)\right\|} \leq C \frac{\varphi(n-k)}{\varphi(k)} .
$$

The right-hand side tends to zero as $k / n \rightarrow 1$ since $\left(e_{n}\right)$ has the LRP. It follows that there exists $\alpha<1$ such that $\left\|G_{k}(x)\right\| \leq C$ for all $k \geq \alpha n$. By iteration $m$ times, where $\alpha^{m} \leq 1 / 2$, we get

$$
\left\|G_{k}(x)\right\| \leq C \quad \text { for all } k \geq n / 2 .
$$

Fix $1 \leq k \leq n$. Let $A:=\{\varrho(1), \ldots, \varrho(k)\}$ and let $B:=\{\varrho(k+1), \ldots, \varrho(2 k)\}$. Choose $D>F$ with $|D|=k$, and let $\varepsilon>0$. Consider

$$
y:=\sum_{i \in F \backslash A} a_{i} e_{i}+\left(\left|a_{\varrho(k)}\right|+\varepsilon\right)\left(\sum_{i \in D} e_{i}\right) .
$$


Then

(3.19) $\sigma_{k}(y) \leq\left\|x+\left(\left|a_{\varrho(k)}\right|+\varepsilon\right)\left(\sum_{i \in D} e_{i}\right)\right\| \leq 1+\left(\left|a_{\varrho(k)}\right|+\varepsilon\right) \varphi(k)$.

Since $\left(e_{i}\right)$ is semi-greedy there exist scalars $c_{i}(i \in D)$ such that

$$
\left\|\sum_{i \in F \backslash A} a_{i} e_{i}+\sum_{i \in D} c_{i} e_{i}\right\| \leq C \sigma_{k}(y) .
$$

Since $F<D,\left(e_{i}\right)$ is a Schauder basis, and $\varepsilon>0$ is arbitrary, (3.19) and (3.20) yield

$$
\left\|\sum_{i \in F \backslash A} a_{i} e_{i}\right\| \leq C\left(1+\left|a_{\varrho(k)}\right| \varphi(k)\right) .
$$

Hence

$$
\left\|G_{k}(x)\right\|=\left\|\sum_{i \in A} a_{i} e_{i}\right\| \leq C\left(1+\left|a_{\varrho(k)}\right| \varphi(k)\right) .
$$

Let $z:=x-\sum_{i \in A} a_{i} e_{i}$. Then $\sigma_{k}(z) \leq\|x\| \leq 1$. Since $\left(e_{i}\right)$ is semi-greedy there exist scalars $\left(c_{i}\right)(i \in B)$ with $\left\|z-\sum_{i \in B} c_{i} e_{i}\right\| \leq C$. Hence

$$
\left\|\sum_{i \in A} a_{i} e_{i}+\sum_{i \in B} c_{i} e_{i}\right\|=\left\|x-\left(z-\sum_{i \in B} c_{i} e_{i}\right)\right\| \leq C .
$$

Let $E:=\left\{i \in B:\left|c_{i}\right| \geq\left|a_{\varrho(k)}\right|\right\}$. Then

$$
\sum_{i \in A} a_{i} e_{i}+\sum_{i \in E} c_{i} e_{i}=G_{m}\left(\sum_{i \in A} a_{i} e_{i}+\sum_{i \in B} c_{i} e_{i}\right)
$$

for some $k \leq m \leq 2 k$. So (3.18) and (3.22) yield

$$
\left\|\sum_{i \in A} a_{i} e_{i}+\sum_{i \in E} c_{i} e_{i}\right\| \leq C .
$$

On the other hand, Remark 3.4 yields

$$
\left|a_{\varrho(k)}\right| \varphi(k) \leq C\left\|\sum_{i \in A} a_{i} e_{i}+\sum_{i \in E} c_{i} e_{i}\right\| .
$$

Finally, combining (3.21), (3.23), and (3.24), we get $\left\|G_{k}(x)\right\| \leq C$.

4. Thresholding-bounded bases. Let $\left(e_{n}\right)$ be a semi-normalized basis for $X$. For $a \geq 0$, the thresholding operator $\mathcal{G}_{a}$ is defined as follows:

$$
\mathcal{G}_{a}(x)=\sum_{\left|e_{n}^{*}(x)\right| \geq a} e_{n}^{*}(x) e_{n} .
$$

We shall also use the notation $\mathcal{G}_{a} x$ for $\mathcal{G}_{a}(x)$. Suppose that there exist $a_{0}>0$ and $K$ such that $\left\|\mathcal{G}_{a_{0}}(x)\right\| \leq K\|x\|$ for all $x \in X$. This implies, by scaling, that $\left\|\mathcal{G}_{a}(x)\right\| \leq K\|x\|$ for all $a>0$ and for all $x \in X$, i.e. that $\left(e_{n}\right)$ is a quasi-greedy basis with quasi-greedy constant $K$. 
To obtain a new class of bases, we consider (following Elton $[5,16]$ ) boundedness of $\mathcal{G}_{a}$ on the set $Q$ of vectors whose coefficient sequences belong to the unit ball of $\ell_{\infty}$ :

$$
Q:=\left\{\sum_{n=1}^{\infty} a_{n} e_{n} \in X:\left|a_{n}\right| \leq 1\right\} .
$$

Let $0<a \leq 1$ and suppose that there exists a constant $C<\infty$ such that

$$
\left\|\mathcal{G}_{a}(x)\right\| \leq C\|x\|
$$

for all $x \in Q$. Let $\theta(a)$ be the least constant $C$ such that (4.25) holds. If there is no such constant $C$, set $\theta(a)=\infty$.

Proposition 4.1. Let $\left(e_{n}\right)$ be a semi-normalized basis for a Banach space $X$.

(i) If $\left(e_{n}\right)$ is a normalized basis, then

$$
\max \left|e_{n}^{*}(x)\right| \leq \theta(1)\|x\| \quad(x \in X) .
$$

(ii) If $0<a \leq b \leq 1$, then $\theta(b) \leq \theta(a)$.

(iii) If $\theta(a)<\infty$ for some $a<1$, then there exist positive constants $C_{1}$ and $b$ such that $\theta(t)<C_{1} t^{-b}$ for all $t \leq 1$.

Proof. (i) By scaling, we may assume that $\max \left|e_{n}^{*}(x)\right|=1$. The result is then clear (from the definition of $\theta(1)$ ) when $\max \left|e_{n}^{*}(x)\right|$ is attained uniquely. By perturbing the basis coefficients of $x$ slightly we can assume that this is the case.

(ii) This follows from the identity

$$
\mathcal{G}_{b}(x)=(b / a) \mathcal{G}_{a}((a / b) x) \quad(x \in Q) .
$$

(iii) Note that, for $k \geq 1$, we have

$$
\mathcal{G}_{a^{k+1}}(x)=\mathcal{G}_{a^{k}}(x)+a^{k} \mathcal{G}_{a}\left(a^{-k}\left(x-\mathcal{G}_{a^{k}}(x)\right)\right) .
$$

Hence by the triangle inequality

$$
\theta\left(a^{k+1}\right) \leq \theta\left(a^{k}\right)+\theta(a)\left(1+\theta\left(a^{k}\right)\right) \leq 3 \theta(a) \theta\left(a^{k}\right) .
$$

It follows that $\theta(t)<C_{1} t^{-b}$ for some positive constants $C_{1}$ and $b$.

We say that $\left(e_{n}\right)$ is thresholding-bounded if $\theta(a)<\infty$ for all $a>0$ (equivalently, for some $a<1$ ).

The next two propositions are "localized" versions of Lemmas 2.1 and 2.2. They will be used in Section 8 below.

Proposition 4.2. Suppose that $\left(e_{n}\right)$ is a thresholding-bounded basis for $X$. Let $\sigma$ be a finite subset of $\mathbb{N}$. Then

$$
\left\|\sum_{n \in \sigma} a_{n} e_{n}\right\| \leq 2 \theta(1) \max \left|a_{n}\right|\left\|\sum_{n \in \sigma} e_{n}\right\|
$$

for all real scalars $\left(a_{n}\right)$. 
Proof. We have

$$
\begin{aligned}
\left\|\sum_{n \in \sigma} a_{n} e_{n}\right\| & \leq \max \left|a_{n}\right| \max _{ \pm}\left\|\sum_{n \in \sigma} \pm e_{n}\right\| \quad \text { (by convexity) } \\
& \leq 2 \max \left|a_{n}\right| \max _{\tau \subseteq \sigma}\left\|\sum_{n \in \tau} e_{n}\right\| \leq 2 \theta(1) \max \left|a_{n}\right|\left\|\sum_{n \in \sigma} e_{n}\right\| .
\end{aligned}
$$

Proposition 4.3. Suppose that $\left(e_{n}\right)$ is a thresholding-bounded basis for $X$. Then, for every $m \geq 1$ and $x \in Q$ with greedy ordering $\varrho$, we have

$$
\left|e_{\varrho(m)}^{*}(x)\right||| \sum_{k=1}^{m}\left(\operatorname{sgn} e_{\varrho(k)}^{*}(x)\right) e_{\varrho(k)}\left\|\leq 2 \theta\left(\left|e_{\varrho(m)}^{*}(x)\right|\right)\right\| x \| .
$$

Proof. Let $a_{j}=e_{j}^{*}(x), \varepsilon_{j}=\operatorname{sgn} a_{j}$, and put $1 /\left|a_{0}\right|=0$. Then

$$
\begin{aligned}
\left|a_{\varrho(m)}\right||| \sum_{j=1}^{m} \varepsilon_{\varrho(j)} e_{\varrho(j)} \| & =\left|a_{\varrho(m)}\right|\left\|\sum_{j=1}^{m}\left(\frac{1}{\left|a_{\varrho(j)}\right|}-\frac{1}{\left|a_{\varrho(j-1)}\right|}\right) \sum_{k=j}^{m} a_{\varrho(k)} e_{\varrho(k)}\right\| \\
& \leq \max _{1 \leq j \leq m}\left\|\sum_{k=j}^{m} a_{\varrho(k)} e_{\varrho(k)}\right\| \leq 2 \theta\left(\left|a_{\varrho(m)}\right|\right)\|x\| .
\end{aligned}
$$

A similar argument gives the following.

Proposition 4.4. Suppose that $\left(e_{n}\right)$ is a thresholding-bounded basis for $X$. Then, for every finite $\sigma \subset \mathbb{N}$ and for all real scalars $\left(a_{n}\right)$ with $\sup \left|a_{n}\right|$ $\leq 1$, we have

$$
\min _{n \in \sigma}\left|a_{n}\right|\left\|\sum_{n \in \sigma}\left(\operatorname{sgn} a_{n}\right) e_{n}\right\| \leq\left(1+\theta\left(\min _{n \in \sigma}\left|a_{n}\right|\right)\right)\left\|\sum_{n \in \sigma} a_{n} e_{n}\right\| .
$$

Note that every quasi-greedy basis is thresholding-bounded and that a thresholding-bounded basis is quasi-greedy with constant $K$ if and only if $\sup _{a>0} \theta(a)=K$.

Let us recall a notion introduced by Elton (see [5, 16]). A semi-normalized basis $\left(e_{n}\right)$ in a Banach space $X$ is called nearly unconditional if, for every $0<$ $a \leq 1$, there exists a constant $\phi(a)$ such that for every $x=\sum_{n=1}^{\infty} e_{n}^{*}(x) e_{n} \in$ $Q$, and for every $A \subseteq\left\{n \in \mathbb{N}:\left|e_{n}^{*}(x)\right| \geq a\right\}$, we have

$$
\left\|\sum_{n \in A} a_{n} e_{n}\right\| \leq \phi(a)\|x\|
$$

Note that $\left(e_{n}\right)$ is unconditional if and only if $\sup _{a>0} \phi(a)<\infty$. Clearly, we have the implication:

$$
\text { nearly unconditional } \Rightarrow \text { thresholding-bounded. }
$$

Surprisingly, the converse implication also holds. 
Proposition 4.5. Every thresholding-bounded basis is nearly unconditional. Moreover,

$$
\phi(a) \leq \frac{4 \theta(1) \theta(a)}{a} \quad(0<a \leq 1) .
$$

Proof. Fix $0<a \leq 1$ and $x \in Q$. Set $\sigma(a):=\left\{i \in \mathbb{N}:\left|e_{i}^{*}(x)\right| \geq a\right\}$, and suppose that $A \subseteq \sigma(a)$. Then

$$
\left\|\sum_{i \in A} e_{i}^{*}(x) e_{i}\right\| \leq \max _{ \pm}\left\|\sum_{i \in \sigma(a)} \pm e_{i}\right\| \leq 2 \theta(1)\left\|\sum_{i \in \sigma(a)}\left(\operatorname{sgn} e_{i}^{*}(x)\right) e_{i}\right\|
$$

(by the same argument used to prove Proposition 4.2)

$$
\leq 4 \theta(1) \frac{\theta(a)}{a}\|x\|
$$

by Proposition 4.3. The estimate for $\phi(a)$ follows.

We conclude this section with an example of a thresholding-bounded basis that is not quasi-greedy. The construction uses the following simple sufficient condition for thresholding-boundedness. Recall first the definition (see also $(6.38)$ below) of the weak- $\ell_{1}$ quasi-norm:

$$
\left\|\left(a_{n}\right)\right\|_{1, \infty}=\sup n a_{n}^{*}
$$

where $\left(a_{n}^{*}\right)$ is the nonincreasing rearrangement of $\left(\left|a_{n}\right|\right)$.

Lemma 4.6. Let $\left(e_{n}\right)$ be a normalized basis of a Banach space $X$ such that

$$
\left\|\sum_{i=1}^{\infty} a_{i} e_{i}\right\| \geq c\left\|\left(a_{i}\right)\right\|_{1, \infty}
$$

for all real scalars $\left(a_{i}\right)$, where $c$ is a constant. Then $\left(e_{n}\right)$ is thresholdingbounded and $\theta(a) \leq(c a)^{-1}$ for $0<a \leq 1$.

Proof. Suppose that $x \in Q$. Then

$$
\left\|\mathcal{G}_{a}(x)\right\| \leq\left|\left\{i:\left|e_{i}^{*}(x)\right| \geq a\right\}\right| \leq \frac{1}{a}\left\|\left(e_{i}^{*}(x)\right)\right\|_{1, \infty} \leq \frac{1}{c a}\|x\| .
$$

Hence $\theta(a) \leq(c a)^{-1}$.

We recall the definition of the dyadic Hardy space $H_{1}$. Let $\left(h_{n}\right)_{n=1}^{\infty}$ be the dyadic Haar system on $[0,1]$ normalized in $L_{1}$. The norm in $H_{1}$ is given as follows:

$$
\left\|\sum_{n=1}^{\infty} a_{n} h_{n}\right\|=\int_{0}^{1}\left(\sum_{n=1}^{\infty} a_{n}^{2} h_{n}^{2}\right)^{1 / 2} d x .
$$

Clearly, $\left(h_{n}\right)$ is a normalized 1-unconditional basis for $H_{1}$.

Lemma 4.7. There exists a constant $C$ such that for every $N \geq 1$ there exist integers $n_{1}, \ldots, n_{2 N}$ and a normalized 1-unconditional basis $\left(e_{i}\right)_{i=1}^{P}$ 
(where $\left.P:=P(N)=\sum_{i=1}^{2 N} n_{i}\right)$ of a finite-dimensional normed space $\left(\mathbb{R}^{P}\right.$, $\left.\|\cdot\|_{N}\right)$, satisfying the following:

(a) For all real scalars $\left(a_{i}\right)_{i=1}^{P}$, we have

$$
\left\|\sum_{i=1}^{P} a_{i} e_{i}\right\|_{N} \geq \frac{1}{C}\left\|\left(a_{i}\right)\right\|_{1, \infty} .
$$

(b) For all real scalars $\left(b_{i}\right)_{i=1}^{2 N}$, we have

$$
\frac{1}{C}\left(\sum_{i=1}^{2 N} b_{i}^{2}\right)^{1 / 2} \leq\left\|\sum_{i=1}^{2 N} b_{i} f_{i}\right\|_{N} \leq C\left(\sum_{i=1}^{2 N} b_{i}^{2}\right)^{1 / 2}
$$

where

$$
f_{i}=\frac{1}{n_{i}}\left(e_{n_{1}+\ldots+n_{i-1}+1}+\ldots+e_{n_{1}+\ldots+n_{i}}\right) .
$$

(c) $n_{1}<n_{3}<\ldots<n_{2 N-1}<n_{2}<n_{4}<\ldots<n_{2 N}$.

Proof. We take the vectors $\left(e_{j}\right)$ for

$$
n_{1}+\ldots+n_{i-1}+1<j \leq n_{1}+\ldots+n_{i}
$$

to be all the Haar functions on a certain level of the $L_{1}$-normalized Haar system on $[0,1]$. It is known that consecutive levels of the dyadic Hardy space $H_{1}$ satisfy (a) and (b). To ensure that (c) is satisfied, we simply rearrange the levels. Since the Haar system is a 1-unconditional basis of $H_{1}$, every rearrangement of the levels is a 1-unconditional basis satisfying (a) and (b).

ExAMPLE 4.8. There exists a reflexive Banach space $X$ with a thresholding-bounded basis that is not quasi-greedy. To see this, recall from [8] the following expression for the norm $\|\cdot\|_{J}$ of the James space $J$ :

$$
\left\|\sum a_{i} e_{i}\right\|_{J}=\sup \left(\sum_{j=1}^{k}\left(\sum_{i=m_{j-1}+1}^{m_{j}} a_{i}\right)^{2}\right)^{1 / 2},
$$

where the supremum is taken over all $k \geq 1$ and all $0=m_{0}<m_{1}<\ldots$ $<m_{k}$. Fix $N \geq 1$ and let $\left(f_{i}\right)_{i=1}^{2 N}$ be defined as in Lemma 4.7. Note that

$$
\left\|f_{1}+f_{3}+\ldots+f_{2 N-1}\right\|_{J}=N
$$

and that

$$
\left\|f_{1}-f_{2}+f_{3}-f_{4}+\ldots+f_{2 N-1}-f_{2 N}\right\|_{J} \leq 2 \sqrt{N} .
$$

Define the norm $|x|_{N}:=\max \left(\|x\|_{N},\|x\|_{J}\right)$. Consider

$$
x=f_{1}-f_{2}+f_{3}-f_{4}+\ldots+f_{2 N-1}-f_{2 N} \quad \text { and } \quad a=1 / n_{2 N-1} .
$$

Then $x \in Q$; by (4.27) and condition (b) of Lemma 4.7, we have

$$
|x|_{N} \leq 2 C \sqrt{N}
$$


By (4.26) and condition (c) of Lemma 4.7, we have

$$
\left|\mathcal{G}_{a}(x)\right|_{N}=\left|f_{1}+f_{3}+\ldots+f_{2 N-1}\right|_{N}=N .
$$

Hence $\theta(a) \geq(2 C)^{-1} \sqrt{N}$. Thus, for each $N$ we have constructed a finitedimensional normed space $\left(F_{N},|\cdot|_{N}\right)$ with a monotone basis $\left(e_{i}\right)$ such that

$$
\left|\sum a_{i} e_{i}\right|_{N} \geq \frac{1}{C}\left\|\left(a_{i}\right)\right\|_{1, \infty}
$$

and

$$
\sup _{a>0} \theta(a) \geq \frac{1}{2 C} \sqrt{N}
$$

Let $X=\left(\sum_{N=1}^{\infty} \oplus F_{N}\right)_{2}$ and let $\left(e_{n}\right)$ be the natural basis obtained by concatenating the bases of the $F_{N}$ 's. For $x=\left(x_{N}\right) \in Q$, using Lemma 4.6 and (4.28) we get

$$
\left\|\mathcal{G}_{a}(x)\right\|=\left(\sum_{N=1}^{\infty}\left|\mathcal{G}_{a}\left(x_{N}\right)\right|_{N}^{2}\right)^{1 / 2} \leq \frac{C}{a}\left(\sum_{N=1}^{\infty}\left|x_{N}\right|_{N}^{2}\right)^{1 / 2}=\frac{C}{a}\|x\| .
$$

Hence $\left(e_{n}\right)$ is a thresholding-bounded basis for $X$. However, by (4.29), $\theta(a) \rightarrow \infty$ as $a \rightarrow 0$. Thus, $\left(e_{n}\right)$ is not quasi-greedy.

5. Subsequences of weakly null sequences. The motivation for the results of this section is the following theorem of Elton [5] (paraphrased slightly to suit our purposes).

THEOREM 5.1. Let $\left(e_{n}\right)$ be a semi-normalized weakly null sequence in a Banach space $X$. Then $\left(e_{n}\right)$ has a thresholding-bounded basic subsequence satisfying

$$
\phi(a) \leq C \log (1+1 / a)
$$

for some absolute constant $C$.

REMARK 5.2. The estimate for $\phi(a)$, while not hitherto explicitly stated anywhere, follows from the proof of Elton's theorem presented in [16]. In its usual formulation, Elton's theorem asserts the existence of a subsequence that is nearly unconditional. However, by Proposition 4.5, this is equivalent to the above.

For Banach spaces which are "far from $c_{0}$ " (in a sense made precise below) we can improve this result significantly by showing the existence of a quasi-greedy subsequence.

To that end let us recall the notion of spreading model (see e.g. [1]). Let $\left(e_{i}\right)$ be a semi-normalized basic sequence in a Banach space $X$ and let $\left(s_{i}\right)$ be a basis for a Banach space $(Y,|\cdot|)$. Then $\left(s_{i}\right)$ is said to be a spreading 
model for $\left(e_{i}\right)$ if, for all $k \geq 1$ and for all real scalars $a_{1}, \ldots, a_{k}$, we have

$$
\left|\sum_{i=1}^{k} a_{i} s_{i}\right|=\lim _{\substack{n_{1}<\ldots<n_{k} \\ n_{1} \rightarrow \infty}}\left\|\sum_{i=1}^{k} a_{i} x_{n_{i}}\right\| .
$$

It is known that every normalized basic sequence has a subsequence with a spreading model, and that if the basic sequence is weakly null, then the spreading model is monotone and 2-unconditional.

Proposition 5.3. Let $\left(e_{i}\right)$ be a semi-normalized basic sequence in a $B a$ nach space $X$ which has spreading model $\left(s_{i}\right)$. Suppose that

$$
g(n):=\left|\sum_{i=1}^{n} s_{i}\right| \rightarrow \infty \quad \text { as } n \rightarrow \infty .
$$

Then $\left(e_{n}\right)$ has a democratic subsequence. Moreover, if $\left(e_{n}\right)$ is weakly null then we may take the democratic constant of the subsequence to be $\Delta \leq 1+\varepsilon$ for any given $\varepsilon>0$.

Proof. Since $\left(s_{i}\right)$ is (by definition) democratic, by using the condition $g(n) \rightarrow \infty$ it is easy to construct the desired democratic subsequence of $\left(e_{i}\right)$. To avoid repetition, we refer to Theorem 5.4 below for a similar, but more complicated, argument. To get $\Delta \leq 1+\varepsilon$ in the weakly null case, we use the fact that $\left(s_{i}\right)$ is monotone when $\left(e_{i}\right)$ is weakly null.

To obtain the main result of this section we need the following result, which is stated without proof in the Introduction of [15] (see also [1] for the proof). Given $\varepsilon>0$, every semi-normalized weakly null sequence has a subsequence $\left(e_{n}\right)$ which is $(2+\varepsilon)$-Schreier-unconditional, i.e. such that

$$
\left\|P_{A} x\right\| \leq(2+\varepsilon)\|x\|
$$

for every $x \in\left[e_{n}\right]$ and for every finite $A \subset \mathbb{N}$ satisfying $|A| \leq \min A$. (Here $P_{A} x:=x \chi_{A}$ denotes the projection onto $A$.)

THEOREM 5.4. Let $\left(e_{n}\right)$ be a semi-normalized weakly null basic sequence in a Banach space $X$ with spreading model $\left(s_{n}\right)$. Suppose that

$$
g(n):=\left|\sum_{i=1}^{n} s_{i}\right| \rightarrow \infty \quad \text { as } n \rightarrow \infty .
$$

Then, given $\varepsilon>0,\left(e_{n}\right)$ has a quasi-greedy subsequence with quasi-greedy constant $K \leq 3+\varepsilon$.

Proof. By passing to a subsequence and rescaling, we may assume that $\left(e_{n}\right)$ is a normalized basic sequence, with basis constant at most $1+\varepsilon / 4$. Choose an increasing sequence $\left(n_{k}\right)$ such that $g\left(n_{k}\right)>24 k / \varepsilon$. Using Schreierunconditionality, and by passing to further subsequences, we may assume 
that $\left(e_{n}\right)$ satisfies the following: for every $k \geq 1$ and for every $A \subset \mathbb{N}$ with $|A| \leq n_{k}$ and $\min A \geq k$, we have

$$
\frac{1}{2}\left\|\sum_{i=1}^{|A|} a_{i} s_{i}\right\| \leq\left\|\sum_{i \in A} a_{i} e_{i}\right\| \leq 2\left\|\sum_{i=1}^{|A|} a_{i} s_{i}\right\|
$$

and

$$
\left\|P_{A} x\right\| \leq(2+\varepsilon / 4)\|x\| .
$$

Suppose that $x=\sum_{i=1}^{\infty} x_{i} e_{i}$ and $\|x\|=1$. Suppose that $a>\varepsilon / k$, where $k \geq 2$. Define

$$
D:=\left\{i \geq k:\left|x_{i}\right| \geq a\right\} \quad \text { and } \quad E:=\left\{i<k:\left|x_{i}\right| \geq a\right\} .
$$

Suppose, to derive a contradiction, that $|D| \geq n_{k}$. Choose $A \subseteq D$ with $|A|=n_{k}$. Then by (5.31),

$$
\left\|P_{A} x\right\| \leq(2+\varepsilon / 4)\|x\|=2+\varepsilon / 4 .
$$

On the other hand,

$$
\begin{aligned}
\left\|P_{A} x\right\| & =\left\|\sum_{i \in A} e_{i}^{*}(x) e_{i}\right\| \geq \frac{1}{2}\left|\sum_{i=1}^{|A|} e_{i}^{*}(x) s_{i}\right| \quad(\text { by }(5.30)) \\
& \left.\geq \frac{1}{4} \min \left\{\left|e_{i}^{*}(x)\right|: i \in A\right\} g(|A|) \quad \text { (by 2-unconditionality of }\left(s_{i}\right)\right) \\
& \geq \frac{1}{4} a g(|A|)>\frac{\varepsilon g\left(n_{k}\right)}{8 k}>3,
\end{aligned}
$$

which is the desired contradiction. Hence $|D|<n_{k}$; in particular,

$$
\left\|P_{D} x\right\| \leq(2+\varepsilon / 4)\|x\|=2+\varepsilon / 4 .
$$

First suppose $a>\varepsilon / 2$, i.e. $k=2$. Then, since the basis constant of $\left(e_{i}\right)$ is at most $1+\varepsilon / 4$,

$$
\left\|\mathcal{G}_{a} x\right\| \leq\left|a_{1}\right|+\left\|P_{D} x\right\| \leq(1+\varepsilon / 4)+(2+\varepsilon / 4)<3+\varepsilon .
$$

Now suppose that $a \leq \varepsilon / 2$. Choose $k \geq 2$ such that

$$
1 / k<2 a / \varepsilon \leq 1 /(k-1) .
$$

By the triangle inequality,

$$
\left\|P_{E} x\right\| \leq\left\|\sum_{i=1}^{k-1} e_{i}^{*}(x) e_{i}\right\|+(k-1) a \leq(1+\varepsilon / 4)\|x\|+\varepsilon / 2=1+3 \varepsilon / 4 .
$$

Combining, we get

$$
\left\|\mathcal{G}_{a} x\right\| \leq\left\|P_{D} x\right\|+\left\|P_{E} x\right\| \leq(2+\varepsilon / 4)+(1+3 \varepsilon / 4)=3+\varepsilon .
$$

By homogeneity, (5.32) and (5.33) prove that $\theta(a) \leq 3+\varepsilon$ for all $a \in(0,1]$, i.e. that $\left(e_{n}\right)$ is quasi-greedy with quasi-greedy constant $K \leq 3+\varepsilon$. 
THEOREM 5.5. Let $\left(e_{n}\right)$ be a semi-normalized democratic weakly null basic sequence in a Banach space. Then $\left(e_{n}\right)$ has an almost greedy subsequence.

Proof. Let $(\varphi(n))$ be the fundamental function. We may suppose that $\left(e_{i}\right)$ has spreading model $\left(s_{i}\right)$. Suppose that $\left(s_{i}\right)$ is not equivalent to the unit vector basis of $c_{0}$. Then, since $\left(s_{i}\right)$ is unconditional, $\left|\sum_{i=1}^{n} s_{i}\right| \rightarrow \infty$ as $n \rightarrow \infty$. Theorem 5.4 now shows that $\left(e_{i}\right)$ has a quasi-greedy, and hence almost greedy, subsequence.

On the other hand, suppose that $\left(s_{i}\right)$ is equivalent to the unit vector basis of $c_{0}$. Then $(\varphi(n))$ is bounded above by $K^{\prime}$, say. Thus, by (2.10),

$$
\left\|\sum_{i=1}^{n} a_{i} e_{i}\right\| \leq 2 \varphi(n) \max \left|a_{i}\right| \leq 2 K^{\prime} \max \left|a_{i}\right| .
$$

Thus, $\left(e_{n}\right)$ is equivalent to the unit vector basis of $c_{0}$, which is greedy.

Corollary 5.6. Suppose that $X$ is a Banach space which does not have $c_{0}$ as a spreading model (e.g., if $X$ has finite cotype; see (3.16) above). Then every semi-normalized weakly null sequence in $X$ has an almost greedy subsequence.

Corollary 5.7. Let $X$ be a Banach space. Then the following are equivalent:

(i) X contains a weakly null sequence with spreading model not equivalent to the unit vector basis of $c_{0}$ or $X$ contains an isomorphic copy of $c_{0}$ or $\ell_{1}$.

(ii) $X$ contains an almost greedy basic sequence.

(iii) $X$ contains a semi-greedy basic sequence.

(iv) $X$ contains a superdemocratic basic sequence.

Proof. (i) $\Rightarrow$ (ii) is immediate from Theorem 5.5 , (ii) $\Rightarrow$ (iii) is Theorem 3.2, and (iii) $\Rightarrow$ (iv) is Proposition 3.3. Suppose that (iv) holds. Let $\left(x_{n}\right)$ be a superdemocratic basic sequence. If $X$ does not contain $\ell_{1}$, then by Rosenthal's $\ell_{1}$ theorem [19], we may assume by passing to a subsequence, setting $y_{n}:=x_{2 n}-x_{2 n-1}$, that $\left(y_{n}\right)_{n=1}^{\infty}$ is a weakly null basic sequence with spreading model $\left(s_{n}\right)$. Clearly, $\left(y_{n}\right)$ is also superdemocratic, and therefore democratic. If $X$ does not contain $c_{0}$, then from the proof of Theorem 5.5 we see that $\left(s_{n}\right)$ is not equivalent to the unit vector basis of $c_{0}$. Thus, (i) holds.

REMARK 5.8. The "original" Tsirelson space [21] does not contain a subspace isomorphic to $c_{0}$ or to $\ell_{1}$ and yet all of its spreading models $\left(s_{i}\right)$ are equivalent to the unit vector basis of $c_{0}$. Thus, this space does not contain any democratic basic sequence. 
6. Existence of democratic bases. Let $X$ be a Banach space with a basis $\left(b_{n}\right)$. By passing to an equivalent norm we may assume that $\left(b_{n}\right)$ is normalized and bimonotone. Let $S$ be a 1-symmetric and 1-unconditional symmetric sequence space with Schauder basis $\left(e_{i}\right)$ (here $e_{i}$ denotes the sequence $\left.\left(\delta_{i j}\right)_{j=1}^{\infty}\right)$. Let $\left(e_{i}^{*}\right)$ be the sequence of biorthogonal functionals in $S^{*}$. Define

$$
f(n):=\left\|e_{1}+\ldots+e_{n}\right\|_{S}
$$

and

$$
g(n):=n / f(n)=\left\|e_{1}^{*}+\ldots+e_{n}^{*}\right\|_{S^{*}} .
$$

We shall assume that $\left(e_{i}\right)$ is not equivalent to the unit vector basis of $c_{0}$. Thus,

$$
f(n) \uparrow \infty \quad \text { as } n \rightarrow \infty .
$$

For $n \geq 1$, let $\sigma_{n}=\left[2^{n-1}, 2^{n}-1\right]$, so that $\left|\sigma_{n}\right|=2^{n-1}$. Let

$$
v_{n}:=\frac{1}{f\left(2^{n-1}\right)} \sum_{k \in \sigma_{n}} e_{k} \quad \text { and } \quad v_{n}^{*}:=\frac{1}{g\left(2^{n-1}\right)} \sum_{k \in \sigma_{n}} e_{k}^{*} .
$$

Let $P$ be the norm-one projection on $S$ defined by

$$
P \xi:=\sum_{n=1}^{\infty}\left\langle\xi, v_{n}^{*}\right\rangle v_{n}
$$

and let $Q=I-P$. Define a norm on $c_{00}$ by

$$
\|\xi\|_{Y}:=\|Q \xi\|_{S}+\left\|\sum_{n=1}^{\infty}\left\langle\xi, v_{n}^{*}\right\rangle b_{n}\right\|_{X}
$$

and then complete to obtain a sequence space $Y$.

Proposition 6.1. Suppose that $\left(b_{n}\right)$ is a bimonotone basis for $X$. Then $\left(e_{n}\right)$ is a Schauder basis for $Y$ such that

$$
\frac{1}{8} \sup _{n} \eta_{n} f(n) \leq\|\xi\|_{Y} \leq 6 \sum_{n=1}^{\infty} \frac{f(n)}{n} \eta_{n}
$$

for all real scalars $\xi=\left(\xi_{n}\right)$ in $c_{00}$, where $\left(\eta_{i}\right)$ is the nonincreasing rearrangement of $\left(\left|\xi_{i}\right|\right)$.

Proof. It is easy to check that the spaces $\left(F_{n}\right)=\left[e_{k}: k \in \sigma_{n}\right]$ form a Schauder decomposition for $Y$ and that each is 3-isomorphic to $\left[e_{k}: k \in \sigma_{n}\right]$ considered as a subspace of $S$. Hence $\left\{e_{k}: k \in \sigma_{n}\right\}$ forms a basis of each $F_{n}$ with uniformly bounded basis constant. Thus $\left(e_{n}\right)$ is a basis of $Y$. For the upper estimate, note that

$$
\left\|\sum_{n=1}^{\infty}\left\langle\xi, v_{n}^{*}\right\rangle b_{n}\right\|_{X} \leq \sum_{n=1}^{\infty} \frac{1}{g\left(2^{n-1}\right)} \sum_{k \in \sigma_{n}}\left|\xi_{k}\right| \leq 2 \sum_{n=1}^{\infty} \frac{f(n)}{n} \eta_{n} .
$$


Also

$$
\begin{aligned}
\|Q \xi\|_{S} & \leq 2\|\xi\|_{S} \leq 2 \sum_{n=1}^{\infty}\left(\eta_{n}-\eta_{n+1}\right) f(n) \quad \text { (by partial summation) } \\
& =2 \eta_{1} f(1)+2 \sum_{n=2}^{\infty} \eta_{n}(f(n)-f(n-1)) \leq 4 \sum_{n=1}^{\infty} \frac{f(n)}{n} \eta_{n}
\end{aligned}
$$

since $f(n)-f(n-1) \leq f(n-1) /(n-1) \leq 2 f(n) / n$. Combining, we get

$$
\|\xi\|_{Y}=\|Q \xi\|_{S}+\left\|\sum_{n=1}^{\infty}\left\langle\xi, v_{n}^{*}\right\rangle b_{n}\right\|_{X} \leq 6 \sum_{n=1}^{\infty} \frac{f(n)}{n} \eta_{n} .
$$

For the lower estimate, suppose that $\|\xi\|_{Y}=1$. For $a>0$, define

$$
A:=\left\{k:\left|\xi_{k}\right|>a\right\} \quad \text { and } \quad B:=\left\{n:\left|\left\langle\xi, v_{n}^{*}\right\rangle\right|<\frac{1}{2} f\left(2^{n-1}\right) a\right\} .
$$

We assume that $|A|=N \geq 1$. Then

$$
1=\|\xi\|_{Y} \geq\|Q \xi\|_{S} \geq \frac{a}{2} f\left(\sum_{n \in B}\left|A \cap \sigma_{n}\right|\right) .
$$

Let $D:=\mathbb{N} \backslash B$. Then

$$
1=\|\xi\|_{Y} \geq\left\|\sum_{n=1}^{\infty}\left\langle\xi, v_{n}^{*}\right\rangle b_{n}\right\|_{X} \geq \frac{1}{2} \max _{n \in D} f\left(2^{n-1}\right) a,
$$

by the bimonotonicity of $\left(b_{n}\right)$. Now (6.35), (6.36), and the fact that $A$ is nonempty imply that $a \leq 2$. Hence there exists a largest positive integer $m$ such that $f\left(2^{m-1}\right) a \leq 2$. Moreover, (6.36) implies that $D \subset\{1, \ldots, m\}$. Hence

$$
\sum_{n \in D}\left|A \cap \sigma_{n}\right| \leq 2^{m}
$$

From this and (6.35) we deduce that

$$
\frac{a}{2} f\left(N-2^{m}\right) \leq \frac{a}{2} f\left(\sum_{n \in B}\left|A \cap \sigma_{n}\right|\right) \leq 1 .
$$

Hence $N \leq 2^{m+1}$ from the choice of $m$. Thus,

$$
a f\left(\left|\left\{k:\left|\xi_{k}\right|>a\right\}\right|\right) \leq a f\left(2^{m+1}\right) \leq 4 a f\left(2^{m-1}\right) \leq 8 .
$$

By homogeneity, we get

$$
\|\xi\|_{Y} \geq \frac{1}{8} \sup _{a>0} a f\left(\left|\left\{k:\left|\xi_{k}\right|>a\right\}\right|\right)=\frac{1}{8} \sup _{n} \eta_{n} f(n)
$$

for all $\xi$.

Theorem 6.2. Suppose that $X$ is a Banach space with a basis which contains a complemented subspace isomorphic to $S$. Then $X$ has a basis 
$\left(e_{n}\right)$ satisfying

$$
\frac{1}{C} \sup _{n} \eta_{n} f(n) \leq\left\|\sum_{n=1}^{\infty} \xi_{n} e_{n}\right\|_{Y} \leq C \sum_{n=1}^{\infty} \frac{f(n)}{n} \eta_{n}
$$

for some constant $C$.

Proof. We may suppose that $X$ has a bimonotone basis $\left(b_{n}\right)$. The space $Y$ constructed above is isomorphic to $X \oplus Q(S)$. By assumption, we have $X \sim Z \oplus S$ for some Banach space $Z$. Thus,

$$
Y \sim X \oplus Q(S) \sim Z \oplus S \oplus Q(S)
$$

By [13, p. 117], $S \sim S \oplus P(S)$. Hence

$$
Y \sim Z \oplus S \oplus P(S) \oplus Q(S) \sim Z \oplus S \oplus S \sim Z \oplus S \sim X
$$

By Proposition $6.1, Y$ has a basis $\left(e_{n}\right)$ with the required property, and hence so does $X$.

To state our next corollary, let us recall the definition of the quasi-norms $\|\cdot\|_{p, 1}$ and $\|\cdot\|_{p, \infty}$ of the Lorentz sequence spaces $\ell_{p, 1}$ and $\ell_{p, \infty}(1 \leq p<\infty)$; here $\left(a_{n}^{*}\right)$ denotes the nonincreasing rearrangement of the sequence $\left(\left|a_{n}\right|\right)$ :

$$
\left\|\left(a_{n}\right)\right\|_{p, 1}=\sum_{n=1}^{\infty} a_{n}^{*} n^{1 / p-1} \quad \text { and } \quad\left\|\left(a_{n}\right)\right\|_{p, \infty}=\sup n^{1 / p} a_{n}^{*} .
$$

Corollary 6.3. Let $1 \leq p<\infty$. Suppose that $X$ is a Banach space with a basis which contains a complemented subspace isomorphic to $\ell_{p}$. Then $X$ has a superdemocratic basis $\left(e_{n}\right)$ satisfying

$$
\frac{1}{C}\left\|\left(a_{i}\right)\right\|_{p, \infty} \leq\left\|\sum_{i=1}^{\infty} a_{i} e_{i}\right\| \leq C\left\|\left(a_{i}\right)\right\|_{p, 1}
$$

for some constant $C$. In particular,

$$
\left\|\sum_{n \in A} \pm e_{n}\right\| \sim|A|^{1 / p}
$$

for all choices of signs and for all finite $A \subset \mathbb{N}$.

Proof. Apply Theorem 6.2 with $S=\ell_{p}$, so that $f(n)=n^{1 / p}$.

REMARK 6.4. Corollary 6.3 implies a theorem of Wojtaszczyk [22, Theorem 4.5] on the existence of a normalized basis of $X$ that is $q$-Besselian for all $q>p$. (Recall that a basis is $q$-Besselian if it satisfies a lower estimate $\left.\left\|\sum_{i=1}^{\infty} a_{i} e_{i}\right\| \geq c\left\|\left(a_{i}\right)\right\|_{q}\right)$

Corollary 6.5. Suppose that $S$ has finite cotype. Let $X$ be a Banach space with a basis which contains a complemented subspace isomorphic to $S$. Then $X$ has a superdemocratic basis with fundamental function equivalent to $(f(n))$. 
Proof. Consider the basis $\left(e_{i}\right)$ of $X$ satisfying (6.37). Suppose that $S$ has cotype $q$ with constant $C_{q}$. Then by Proposition 3.5,

$$
f(m n) \geq\left(1 / C_{q}\right) m^{1 / q} f(n) .
$$

Hence, for all $A \subset \mathbb{N}$ with $|A|=n$, we have

$$
\frac{f(n)}{C} \leq\left\|\sum_{i \in A} \pm e_{i}\right\| \leq C \sum_{k=1}^{n} \frac{f(k)}{k} \leq C C_{q} \frac{f(n)}{n^{1 / q}} \sum_{k=1}^{n} k^{1 / q-1} \leq C^{\prime} f(n) .
$$

So $\left(e_{i}\right)$ is superdemocratic with fundamental function equivalent to $(f(n))$.

7. Existence of quasi-greedy bases. We continue to use the notation introduced in Section 6 . We start with the case $S=\ell_{1}$, which requires special treatment.

Theorem 7.1. Suppose that $\left(b_{n}\right)$ is a basis for $X$ and that $S=\ell_{1}$. Then $\left(e_{n}\right)$ is a quasi-greedy basis for $Y$.

Proof. We may assume as above that $\left(b_{n}\right)$ is a normalized bimonotone basis. It is convenient to introduce the following notation. Fix $a>0$. Then, for $x \in \mathbb{R}$, define $x^{a}$ as follows:

$$
x^{a}:= \begin{cases}x & \text { for }|x| \geq a, \\ 0 & \text { for }|x| \leq a .\end{cases}
$$

For $\xi=\left(\xi_{n}\right) \in c_{00}$, let $\xi^{a}=\left(\xi_{n}^{a}\right)\left(=\mathcal{G}_{a} \xi\right)$. Note that

$$
\begin{gathered}
\|\xi\|_{Y}=\|Q \xi\|_{1}+\left\|\sum_{n=1}^{\infty}\left(\sum_{k \in \sigma_{n}} \xi_{k}\right) b_{n}\right\|_{X}, \\
\left\|\mathcal{G}_{a} \xi\right\|_{Y}=\left\|Q \xi^{a}\right\|_{1}+\left\|\sum_{n=1}^{\infty}\left(\sum_{k \in \sigma_{n}} \xi_{k}^{a}\right) b_{n}\right\|_{X} .
\end{gathered}
$$

Suppose that $\|\xi\|_{Y}=1$. Since $\left(b_{n}\right)$ is bimonotone, we have

$$
\left|\sum_{k \in \sigma_{n}} \xi_{k}\right| \leq 1 \quad(n \geq 1)
$$

Moreover, (7.39) clearly implies that $\mathcal{G}_{a} \xi=0$ if $a>1$. So we shall assume that $a \leq 1$. Let $N$ be the smallest positive integer for which $a>2 /\left|\sigma_{N}\right|$. Note that $N \geq 2$, since $a \leq 1$. For $n \geq N$, we have

$$
\frac{1}{\left|\sigma_{n}\right|}\left|\sum_{k \in \sigma_{n}} \xi_{k}\right|<\frac{a}{2}
$$

Let $E_{n}$ denote the projection onto $\sigma_{n}$, i.e. $E_{n} \xi=\xi \chi_{\sigma_{n}}$. Then

$$
\left|\sum_{k \in \sigma_{n}} \xi_{k}^{a}\right| \leq 2 \sum_{k \in \sigma_{n}}\left|\xi_{k}-\frac{1}{\left|\sigma_{n}\right|} \sum_{k \in \sigma_{n}} \xi_{k}\right|=2\left\|E_{n} Q \xi\right\|_{1} \quad(n \geq N) .
$$


Hence, by the triangle inequality,

$$
\left\|\sum_{n=N}^{\infty}\left(\sum_{k \in \sigma_{n}} \xi_{k}^{a}\right) b_{k}\right\|_{X} \leq 2 \sum_{n=N}^{\infty}\left\|E_{n} Q \xi\right\|_{1} \leq 2\|\xi\|_{Y} .
$$

Also

$$
\begin{aligned}
& \left\|\sum_{n=1}^{N-1}\left(\sum_{k \in \sigma_{n}} \xi_{k}^{a}\right) b_{n}\right\|_{X} \\
& \leq\left\|\sum_{n=1}^{N-1}\left(\sum_{k \in \sigma_{n}} \xi_{k}\right) b_{n}\right\|_{X}+\left\|\sum_{n=1}^{N-1}\left(\sum_{k \in \sigma_{n}}\left(\xi_{k}^{a}-\xi_{k}\right)\right) b_{n}\right\|_{X} \\
& \leq\|\xi\|_{Y}+\left(\sum_{n=1}^{N-1}\left|\sigma_{n}\right|\right) a \quad\left(\text { since }\left|\xi_{k}^{a}-\xi_{k}\right| \leq a\right) \\
& \leq\|\xi\|_{Y}+2\left|\sigma_{N-1}\right| \frac{2}{\left|\sigma_{N-1}\right|}=\|\xi\|_{Y}+4 .
\end{aligned}
$$

Combining (7.41) and (7.42), we get

$$
\left\|\sum_{n=1}^{\infty}\left(\sum_{k \in \sigma_{n}} \xi_{k}^{a}\right) b_{n}\right\|_{X} \leq 3\|\xi\|_{Y}+4
$$

Similarly, for all $n \geq N$, we have $\left\|E_{n} \mathcal{G}_{a} \xi\right\|_{1} \leq 2\left\|E_{n} Q \xi\right\|_{1}$, whence

$$
\begin{aligned}
\left\|\left(\sum_{n=N}^{\infty} E_{n}\right) Q \mathcal{G}_{a} \xi\right\|_{1} & \leq\|Q\| \sum_{n=N}^{\infty}\left\|E_{n} \mathcal{G}_{a} \xi\right\|_{1} \\
& \leq 4 \sum_{n=N}^{\infty}\left\|E_{n} Q \xi\right\|_{1} \leq 4\|\xi\|_{Y} .
\end{aligned}
$$

Also

$$
\begin{aligned}
\left\|\left(\sum_{n=1}^{N-1} E_{n}\right) Q \mathcal{G}_{a} \xi\right\|_{1} & \leq\left\|\left(\sum_{n=1}^{N-1} E_{n}\right) Q\left(\xi-\mathcal{G}_{a} \xi\right)\right\|_{1}+\left\|\left(\sum_{n=1}^{N-1} E_{n}\right) Q \xi\right\|_{1} \\
& \leq 2 a\left(\sum_{n=1}^{N-1}\left|\sigma_{n}\right|\right)+\|\xi\|_{Y} \\
& \leq 4 a\left|\sigma_{N-1}\right|+\|\xi\|_{Y} \leq 8+\|\xi\|_{Y} .
\end{aligned}
$$

Combining (7.43) and (7.44), we get

$$
\left\|Q \mathcal{G}_{a} \xi\right\|_{1} \leq 5\|\xi\|_{Y}+8 .
$$

Finally, 


$$
\left\|\mathcal{G}_{a} \xi\right\|_{Y}=\left\|Q \mathcal{G}_{a} \xi\right\|_{1}+\left\|\sum_{n=1}^{\infty}\left(\sum_{k \in \sigma_{n}} \xi_{k}^{a}\right) b_{n}\right\|_{X} \leq 8\|\xi\|_{Y}+12=20\|\xi\|_{Y}
$$

Hence $\left(e_{n}\right)$ is quasi-greedy with quasi-greedy constant $K \leq 20$.

Now we consider the case $S \neq \ell_{1}$. Here we can prove a more general result by dropping the assumption that $S$ is symmetric. Let us say that $\left(e_{n}\right)$ is a good unconditional basis if it satisfies three conditions:

(1) There is a function $f(n) \uparrow \infty$ as $n \rightarrow \infty$ such that $\left\|\sum_{j \in A} e_{j}\right\| \geq f(|A|)$ for all finite $A \subset \mathbb{N}$.

(2) There is a normalized block basic sequence $\left(u_{n}\right)$ with biorthogonal sequence $\left(u_{n}^{*}\right)$ such that

$$
\lim _{n \rightarrow \infty}\left\|u_{n}\right\|_{\infty}=\lim _{n \rightarrow \infty}\left\|u_{n}^{*}\right\|_{\infty}=0 .
$$

(3) The projection

$$
P \xi=\sum\left\langle\xi, u_{n}^{*}\right\rangle u_{n}
$$

is bounded on $S$.

Note that if $S$ has a symmetric basis and if $S$ is neither $c_{0}$ nor $\ell_{1}$, then the basis is good. Both $c_{0}$ and $\ell_{1}$ fail to satisfy condition (2). In particular, the argument of Theorem 7.2 does not work for $S=\ell_{1}$.

THEOREM 7.2. If $S$ has a good unconditional basis and $X$ has a basis then $S \oplus X$ has a quasi-greedy basis.

Proof. We may assume that $X$ has a bimonotone normalized basis $\left(b_{n}\right)$. We suppose $S$ is given as a 1-unconditional sequence space (no longer symmetric) and that

$$
\left\|\sum_{j \in A} e_{j}\right\| \geq f(|A|)
$$

where $f(n) \uparrow \infty$. We suppose further the existence of a normalized block basic sequence $\left(u_{n}\right)$ with dual functionals $\left(u_{n}^{*}\right)$ (also blocked on the same blocks) so that

$$
\delta_{n}:=\max \left(\left\|u_{n}\right\|_{\infty},\left\|u_{n}^{*}\right\|_{\infty}\right) \rightarrow 0
$$

as $n \rightarrow \infty$. We also assume that the projection $P \xi=\sum\left\langle\xi, u_{n}^{*}\right\rangle u_{n}$ is bounded with $\|P\|=\Lambda$. By passing to a subsequence we may suppose that there is an increasing sequence $\left(M_{n}\right)_{n=0}^{\infty}$, with $M_{0}=0$, such that

(i) $u_{n}, u_{n}^{*}$ are supported on $\sigma_{n}=\left(M_{n-1}, M_{n}\right]$.

(ii) $f\left(M_{n}\right)>M_{n-1}$.

(iii) $\delta_{n} M_{n-1}<1$.

(iv) $\delta_{n} \leq \frac{1}{2} \delta_{n-1}$ for $n \geq 2$. 
Let $Q=I-P$. As before we introduce $Y$ as the completion of the norm on $c_{00}$ defined by

$$
\|\xi\|_{Y}=\|Q \xi\|_{S}+\left\|\sum_{n=1}^{\infty}\left\langle\xi, u_{n}^{*}\right\rangle b_{n}\right\|_{X} .
$$

Let $E_{n} \xi=\xi \chi_{\sigma_{n}}$. Since $\left\|E_{1}+\ldots+E_{n}\right\|_{Y}=1$, it follows as before that $\left(e_{n}\right)$ is a basis for $Y$. Let $C$ be the basis constant of $\left(e_{n}\right)$. Note that if $\xi$ is supported on some $\sigma_{n}$, then

$$
\|\xi\|_{Y}=\left\|\xi-\left\langle\xi, u_{n}^{*}\right\rangle u_{n}\right\|_{S}+\left|\left\langle\xi, u_{n}^{*}\right\rangle\right| \leq\|\xi\|_{S}+2\|P \xi\|_{S}
$$

so that

$$
\|\xi\|_{S} \leq\|\xi\|_{Y} \leq(1+2 \Lambda)\|\xi\|_{S}
$$

Note that $Y \sim X \oplus Q(S)$ and hence $Y \oplus P(S) \sim X \oplus S$. Since $\left(u_{n}\right)$ is an unconditional basis for $P(S)$, we need only show that $\left(e_{n}\right)$ is a quasi-greedy basis for $Y$.

Fix $\xi$ with $\|\xi\|_{Y}=(2 C \Lambda)^{-1}$. Note that

$$
\sup \left|e_{i}^{*}(\xi)\right| \leq 1
$$

and hence $\mathcal{G}_{a} \xi=0$ for $a>1$. So we may assume that $0<a \leq 1$. Let $r \geq 0$ be chosen so that $a M_{r}<1$ but $a M_{r+1}>1$. Then, by (7.45),

$$
\begin{aligned}
\left\|\left(E_{1}+\ldots+E_{r}\right)\left(\xi-\mathcal{G}_{a} \xi\right)\right\|_{Y} & \leq(1+2 \Lambda) \sum_{i=1}^{r}\left\|E_{i}\left(\xi-\mathcal{G}_{a} \xi\right)\right\|_{S} \\
& \leq(1+2 \Lambda) a M_{r}<1+2 \Lambda .
\end{aligned}
$$

Thus,

$$
\left\|\left(E_{1}+\ldots+E_{r}\right)\left(\mathcal{G}_{a} \xi\right)\right\|_{Y} \leq 1+2 \Lambda+\|\xi\|_{Y}
$$

Note that

$$
a f\left(M_{r+2}\right)>a M_{r+1}>1 \quad \text { by (ii), }
$$

and that

$$
\delta_{r+2}<1 / M_{r+1}<a \quad \text { by (iii). }
$$

Suppose that $j \geq r+3$. Then, by (7.45),

$$
\left|\left\langle\xi, u_{j}^{*}\right\rangle\right| \leq \Lambda\left\|E_{j} \xi\right\|_{S} \leq \Lambda\left\|E_{j} \xi\right\|_{Y} \leq 2 C \Lambda\|\xi\|_{Y}=1 .
$$

Hence

$$
\left\|E_{j} P \xi\right\|_{\infty} \leq\left\|u_{j}\right\|_{\infty} \leq \delta_{j} \leq \frac{1}{2} \delta_{r+2}<\frac{a}{2}
$$

It follows that $\left|E_{j} Q \xi\right| \geq \frac{1}{2}\left|E_{j} \mathcal{G}_{a} \xi\right|$ (coordinatewise). Hence

$$
\left\|\left(\sum_{j \geq r+3} E_{j}\right)\left(\mathcal{G}_{a} \xi\right)\right\|_{S} \leq 2\|Q \xi\|_{S} \leq 2\|\xi\|_{Y} .
$$


So

$$
\begin{aligned}
\left\|Q\left(\sum_{j \geq r+3} E_{j}\right)\left(\mathcal{G}_{a} \xi\right)\right\|_{S} & \leq\|Q\|\left\|\left(\sum_{j \geq r+3} E_{j}\right)\left(\mathcal{G}_{a} \xi\right)\right\|_{S} \\
& \leq 2(\Lambda+1)\|\xi\|_{Y}
\end{aligned}
$$

Let

$$
L_{j}:=\left|\left\{k \in \sigma_{j}:\left|\xi_{k}\right| \geq a\right\}\right| .
$$

Then, by (7.45) and the 1-unconditionality of $S$,

$$
a f\left(L_{j}\right) \leq\left\|E_{j} \xi\right\|_{S} \leq\|\xi\|_{Y} \leq 1 .
$$

Hence

$$
a f\left(L_{j}\right) \leq 1<a M_{r+1} \leq a f\left(M_{r+2}\right),
$$

which implies that $L_{j}<M_{r+2}$. Thus,

$$
\left|\left\langle\mathcal{G}_{a} \xi, u_{j}^{*}\right\rangle\right| \leq L_{j}\left\|u_{j}^{*}\right\|_{\infty}<M_{r+2} \delta_{j} .
$$

Thus, if $j \geq r+3$, we have, by (iii) and (iv),

$$
\left|\left\langle\mathcal{G}_{a} \xi, u_{j}^{*}\right\rangle\right| \leq M_{r+2} \delta_{r+3} 2^{r+3-j}<2^{r+3-j} .
$$

Together with the triangle inequality, this gives

$$
\left\|\sum_{j=r+3}^{\infty}\left\langle\mathcal{G}_{a} \xi, u_{j}^{*}\right\rangle b_{j}\right\|_{X} \leq 2 .
$$

Combining (7.47) and (7.48), we get

$$
\left\|\left(\sum_{j \geq r+3} E_{j}\right)\left(\mathcal{G}_{a} \xi\right)\right\|_{Y} \leq 2(1+\Lambda)\|\xi\|_{Y}+2 .
$$

We are left to estimate $\left\|E_{j}\left(\mathcal{G}_{a} \xi\right)\right\|_{Y}$ for $j=r+1, r+2$. But then, by (7.45),

$$
\begin{aligned}
\left\|E_{j}\left(\mathcal{G}_{a} \xi\right)\right\|_{Y} & \leq(1+2 \Lambda)\left\|E_{j}\left(\mathcal{G}_{a} \xi\right)\right\|_{S} \leq(1+2 \Lambda)\left\|E_{j} \xi\right\|_{S} \\
& \leq(1+2 \Lambda)\|\xi\|_{Y} .
\end{aligned}
$$

Combining (7.46), (7.49), and (7.50) gives

$$
\left\|\mathcal{G}_{a} \xi\right\|_{Y} \leq 3+2 \Lambda+(5+6 \Lambda)\|\xi\|_{Y}=\left(6 C \Lambda+4 C \Lambda^{2}+5+6 \Lambda\right)\|\xi\|_{Y} .
$$

Hence $\left(e_{n}\right)$ is quasi-greedy with quasi-greedy constant $K \leq 6 C \Lambda+4 C \Lambda^{2}+$ $5+6 \Lambda$.

Corollary 7.3. Suppose that $X$ has a basis and contains a complemented subspace $S$ with a symmetric basis, where $S$ is not isomorphic to $c_{0}$. Then $X$ has a quasi-greedy basis.

Proof. The case $S=\ell_{1}$ is covered by Theorem 7.1. If $S \neq \ell_{1}$ then the basis of $S$ is good. Hence $X \oplus S$ has a quasi-greedy basis. But $X \sim X \oplus S$. 
THEOREM 7.4. Suppose that $X$ has a basis and contains a complemented subspace with a symmetric basis and finite cotype. Then $X$ has an almost greedy basis.

Proof. Combining Corollary 6.5 and Theorem 7.2 (or Theorem 7.1 for the case $S=\ell_{1}$ ) yields a basis that is simultaneously democratic and quasigreedy.

REMARK 7.5. Noncommutative $L_{p}$ spaces do not have an unconditional basis. However, under reasonable assumptions, it is proved in [9] that for $1<p<\infty$ they have a basis (see also [10] for more examples associated with groups). Since noncommutative $L_{p}$ spaces contain complemented copies of $\ell_{p}$, we can apply Theorem 7.4 to obtain the existence of almost greedy bases in these spaces.

REMARK 7.6. Since every closed infinite-dimensional subspace of $\ell_{p}$ contains a complemented copy of $\ell_{p}$, it follows from Theorem 7.4 that every subspace of $\ell_{p}$ with a Schauder basis has an almost greedy basis. On the other hand, it is known that there exist subspaces of $\ell_{p}(p \neq 2)$ with a Schauder basis but without any unconditional basis. See [11] for quantitative finite-dimensional results in this direction.

REMARK 7.7. It is clear that if $\left(b_{n}\right)$ and $\left(b_{n}^{\prime}\right)$ are inequivalent bases for $X$, then the corresponding almost greedy bases $\left(e_{n}\right)$ and $\left(e_{n}^{\prime}\right)$ produced by the construction will be inequivalent. It is known that every Banach space with a basis has infinitely many inequivalent normalized conditional bases [17]. Hence, if $X$ contains a complemented copy of $\ell_{p}$ for some $1 \leq p<\infty$, then $X$ has infinitely many inequivalent almost greedy bases. This yields another proof of the existence of (infinitely many inequivalent) conditional quasi-greedy bases in $\ell_{1}$ or $\ell_{2}$ (cf. $[4,23]$ ).

8. Quasi-greedy bases in $\mathcal{L}_{\infty}$ spaces. This section makes heavy use of a theorem of Grothendieck and related results. So we begin by recalling these important facts.

Let $T: X \rightarrow Y$ be a continuous linear operator between Banach spaces $X$ and $Y$. Then $T$ is called absolutely summing if there exists a constant $C$ such that for all sequences $\left(x_{n}\right)$ in $X$, we have

$$
\sum_{n=1}^{\infty}\left\|T\left(x_{n}\right)\right\| \leq C \sup \left\{\sum_{n=1}^{\infty}\left|x^{*}\left(x_{n}\right)\right|: x^{*} \in X^{*},\left\|x^{*}\right\| \leq 1\right\} .
$$

(Note that the right-hand side of (8.51) equals $C \sup _{\varepsilon_{n}= \pm 1}\left\|\sum_{n=1}^{\infty} \varepsilon_{n} x_{n}\right\|$.) The least such constant $C$ is denoted by $\pi_{1}(T)$. A Banach space $X$ is called a GT space [18] if every bounded linear operator from $X$ to any Hilbert space $H$ is absolutely summing. Grothendieck [7] proved that $L_{1}(\mu)$ spaces are GT spaces. 
Recall that $X$ is an $\mathcal{L}_{\infty}$ space if there exist $\lambda \geq 1$ and a directed net $\left(F_{\alpha}\right)$ of finite-dimensional subspaces of $X$, where each $F_{\alpha}$ is $\lambda$-isomorphic to an $\ell_{\infty}^{n}$ space, such that $X=\overline{\bigcup_{\alpha} F_{\alpha}}$. This class includes every complemented subspace of a $C(K)$ space. If $X$ is an $\mathcal{L}_{\infty}$ space then $X^{*}$ is a GT space (see [18]). Bourgain [2] proved that the dual of the disc algebra is a GT space.

Let $\left(e_{n}\right)$ be a basis for $X$. We say that $\left(e_{n}\right)$ satisfies condition $M_{p}(1 \leq$ $p \leq \infty)$ if there exists a constant $C_{p}$ such that

$$
\left\|\left(x^{*}\left(e_{n}\right)\right)\right\|_{p} \leq C_{p}\left\|x^{*}\right\| \quad\left(x^{*} \in X^{*}\right) .
$$

Note that $M_{p}$ holds if and only if $\left(e_{n}\right)$ is $q$-Hilbertian, i.e., $\left(e_{n}\right)$ satisfies the upper $q$-estimate $(1 / p+1 / q=1)$

$$
\|x\| \leq \frac{1}{C_{p}}\left\|\left(e_{n}^{*}(x)\right)\right\|_{q} \quad(x \in X) .
$$

The basis $\left(e_{n}\right)$ is called Hilbertian if it satisfies $M_{2}$. We note that $C[0,1]$ has a Hilbertian basis [22].

Proposition 8.1. Suppose that $\left(e_{n}\right)$ is a semi-normalized thresholdingbounded Hilbertian basis for a Banach space X. If $X^{*}$ is a GT space, then $\left(e_{n}\right)$ is equivalent to the unit vector basis of $c_{0}$.

Proof. We show that there exists a constant $C$ such that

$$
\sum_{n=1}^{\infty}\left|a_{n}\right| \leq C\left\|\sum_{n=1}^{\infty} a_{n} e_{n}^{*}\right\|
$$

which is equivalent to the result. We may assume without loss of generality that the Hilbertian constant $C_{2}$ equals one. Since $X^{*}$ is a GT space, every bounded linear operator $T$ from $X^{*}$ to $\ell_{2}$ is absolutely summing with $\pi_{1}(T) \leq B\|T\|$ for some absolute constant $B$. Since $\left(e_{n}\right)$ is Hilbertian, the map $X^{*} \rightarrow \ell_{2}$ given by $x^{*} \mapsto\left(x^{*}\left(e_{n}\right)\right)$ is bounded, with operator norm at most $C_{2}=1$, and hence absolutely summing. Thus,

$$
\sum_{n=1}^{\infty}\left|x^{*}\left(e_{n}\right)\right| \leq B \sup _{\varepsilon_{n}= \pm 1}\left\|\sum_{n=1}^{\infty} \varepsilon_{n} x^{*}\left(e_{n}\right) e_{n}^{*}\right\| \quad\left(x^{*} \in X^{*}\right) .
$$

Fix $x^{*}=\sum_{n=1}^{\infty} a_{n} e_{n}^{*} \in\left[e_{n}^{*}\right]$. Choose signs $\left(\varepsilon_{n}\right)$ such that

$$
\left\|\sum_{n=1}^{\infty} a_{n} \varepsilon_{n} e_{n}^{*}\right\| \geq \frac{1}{B} \sum_{n=1}^{\infty}\left|a_{n}\right| .
$$

Choose $x \in X$, with $\|x\|=1$, such that

$$
\sum_{n=1}^{\infty} \varepsilon_{n} a_{n} e_{n}^{*}(x)>\frac{1}{2 B} \sum_{n \in A}\left|a_{n}\right| .
$$


Let $\sigma=\left\{n \in \mathbb{N}:\left|e_{n}^{*}(x)\right|>(4 B)^{-1}\right\}$. Clearly,

$$
\sum_{n \in \sigma} \varepsilon_{n} a_{n} e_{n}^{*}(x)>\frac{1}{4 B} \sum_{n=1}^{\infty}\left|a_{n}\right| .
$$

Set $a=(4 B \theta(1))^{-1}$. Then

$$
\begin{aligned}
\left\|\sum_{n \in \sigma} \varepsilon_{n} e_{n}^{*}(x) e_{n}\right\| & \leq 2 \theta(1)^{2} \| \\
& \quad \sum_{n \in \sigma}\left(\operatorname{sgn} e_{n}^{*}(x)\right) e_{n} \| \\
= & 2 \theta(1)^{2}(4 B)\left(\frac{1}{4 B}\left\|\sum_{n \in \sigma}\left(\operatorname{sgn} e_{n}^{*}(x)\right) e_{n}\right\|\right) \\
\leq & 8 \theta(1)^{2} B(1+\theta(a))\left\|\sum_{n \in \sigma} e_{n}^{*}(x) e_{n}\right\|
\end{aligned}
$$

(by Proposition 4.4 since $(4 B)^{-1} \leq\left|e_{n}^{*}(x)\right| \leq \theta(1)$ for $n \in \sigma$ )

$$
\begin{aligned}
& \leq 8 \theta(1)^{2} B(1+\theta(a)) \theta(a)\|x\| \\
& \quad \quad\left(\text { since } \sum_{n \in \sigma} e_{n}^{*}(x) e_{n}=\theta(1) \mathcal{G}_{a}\left(\theta(1)^{-1} x\right)\right) \\
& =8 \theta(1)^{2} B(1+\theta(a)) \theta(a) .
\end{aligned}
$$

From (8.52), we get

$$
\begin{aligned}
\sum_{n=1}^{\infty}\left|a_{n}\right| & <4 B \sum_{n \in \sigma} \varepsilon_{n} a_{n} e_{n}^{*}(x) \leq 4 B\left\|\sum_{n=1}^{\infty} a_{n} e_{n}^{*}\right\|\left\|\sum_{n \in \sigma} \varepsilon_{n} e_{n}^{*}(x) e_{n}\right\| \\
& \leq C\left\|\sum_{n=1}^{\infty} a_{n} e_{n}^{*}\right\|
\end{aligned}
$$

where $C=32 \theta(1)^{2} B^{2}(1+\theta(a)) \theta(a)$.

Lemma 8.2. Suppose that $\left(e_{n}\right)$ is a normalized thresholding-bounded basis for $X$. Let $\sigma \subset \mathbb{N}$ with $|\sigma|=N \geq 2$. Then, for every choice of signs $\left(\varepsilon_{n}\right)_{n \in \sigma}$, we have

$$
\left\|\sum_{n \in \sigma} \varepsilon_{n} e_{n}^{*}(x) e_{n}\right\| \leq C\left(\log _{2} N\right) \theta(1 / N)\|x\| \quad(x \in X),
$$

where $C=2+8 \theta(1)^{2}(1+\theta(1 / 2))$.

Proof. Suppose that $\|x\|=1 / \theta(1)$, so that $x \in Q$ by (i) of Proposition 4.1. For $k \geq 0$, let

$$
\tau_{k}=\left\{n \in \mathbb{N}: 2^{-k} \leq\left|e_{n}^{*}(x)\right|<2^{1-k}\right\} .
$$

Then, for $k>\left[\log _{2} N\right]$ and $n \in \tau_{k}$, we have $\left|e_{n}^{*}(x)\right| \leq 2 / N$. Hence 


$$
\left\|\sum_{k>\left[\log _{2} N\right]}\left(\sum_{n \in \sigma \cap \tau_{k}} \varepsilon_{n} e_{n}^{*}(x) e_{n}\right)\right\|<\frac{2}{N}|\sigma|=2 \theta(1)\|x\| .
$$

For $k \leq\left[\log _{2} N\right]$, we have

$$
\begin{aligned}
& \left\|\sum_{n \in \sigma \cap \tau_{k}} \varepsilon_{n} e_{n}^{*}(x) e_{n}\right\| \\
& \quad \leq 2 \theta(1)\left\|\sum_{n \in \sigma \cap \tau_{k}} 2^{1-k}\left(\operatorname{sgn} e_{n}^{*}(x)\right) e_{n}\right\| \quad \text { (by Proposition 4.2) } \\
& \quad \leq 2 \theta(1)^{2}\left\|\sum_{n \in \tau_{k}} 2^{1-k}\left(\operatorname{sgn} e_{n}^{*}(x)\right) e_{n}\right\|=4 \theta(1)^{2}\left\|\sum_{n \in \tau_{k}} 2^{-k}\left(\operatorname{sgn} e_{n}^{*}(x)\right) e_{n}\right\| \\
& \quad \leq 4 \theta(1)^{2}(1+\theta(1 / 2))\left\|\sum_{n \in \tau_{k}} e_{n}^{*}(x) e_{n}\right\| \quad \text { (by Proposition 4.4) } \\
& \quad=4 \theta(1)^{2}(1+\theta(1 / 2))\left\|\mathcal{G}_{2^{-k}} x-\mathcal{G}_{2^{1-k}} x\right\| \\
& \quad \leq 8 \theta(1)^{2}(1+\theta(1 / 2)) \theta\left(2^{-k}\right)\|x\| .
\end{aligned}
$$

Hence

$$
\sum_{k=1}^{\left[\log _{2} N\right]}\left\|\sum_{n \in \tau_{k} \cap \sigma} \varepsilon_{n} e_{n}^{*}(x) e_{n}\right\| \leq 8 \theta(1)^{2}(1+\theta(1 / 2))\left(\log _{2} N\right) \theta(1 / N)\|x\| .
$$

Combining (8.53) and (8.54), we get

$$
\left\|\sum_{n \in \sigma} \varepsilon_{n} e_{n}^{*}(x) e_{n}\right\| \leq C\left(\log _{2} N\right) \theta(1 / N)\|x\|
$$

where $C=2+8 \theta(1)^{2}(1+\theta(1 / 2))$.

REMARK 8.3. By duality, we also have

$$
\left\|\sum_{n \in \sigma} \varepsilon_{n} x^{*}\left(e_{n}\right) e_{n}^{*}\right\| \leq C\left(\log _{2} N\right) \theta(1 / N)\|x\| \quad\left(x^{*} \in X^{*}\right) .
$$

LEMma 8.4. Suppose that $\left(e_{n}\right)$ is a normalized thresholding-bounded basis for a Banach space $X$ which satisfies $\theta(a) \leq C a^{-\varepsilon}(0<a \leq 1)$, where $C>0$ and $\varepsilon \in(0,1 / 2)$. Suppose that $X^{*}$ is a $G T$ space and that $M_{p}$ holds for some $p>2$. Then $M_{r}$ holds whenever

$$
\frac{1}{r}<\frac{1}{p}+\frac{1}{2}-\varepsilon
$$

Proof. Let $1 / s=1 / p+1 / 2$. Suppose that $\sigma \subset \mathbb{N}$ with $|\sigma|=N$, and that $\left(\eta_{n}\right)_{n \in \sigma}$ is any fixed choice of signs. Choose $x^{*} \in X^{*}$, with $\left\|x^{*}\right\|=1$, such that

$$
x^{*}\left(\sum_{n \in \sigma} \eta_{n} e_{n}\right)=\left\|\sum_{n \in \sigma} \eta_{n} e_{n}\right\| .
$$


Next consider $T: X^{*} \rightarrow \ell_{2}(\sigma)$ defined as follows:

$$
T y^{*}=\left(y^{*}\left(e_{n}\right)\left|x^{*}\left(e_{n}\right)\right|^{s-1}\right)_{n \in \sigma} \quad\left(y^{*} \in X^{*}\right) .
$$

Then, applying Hölder's inequality and using condition $M_{p}$, we get

$$
\begin{aligned}
\left\|T y^{*}\right\| & =\left(\sum_{n \in \sigma}\left|x^{*}\left(e_{n}\right)\right|^{2 s-2}\left|y^{*}\left(e_{n}\right)\right|^{2}\right)^{1 / 2} \\
& \leq\left(\sum_{n \in \sigma}\left|x^{*}\left(e_{n}\right)\right|^{s}\right)^{1 / 2-1 / p}\left(\sum_{n \in \sigma}\left|y^{*}\left(e_{n}\right)\right|^{p}\right)^{1 / p} \\
& \leq C_{p}\left(\sum_{n \in \sigma}\left|x^{*}\left(e_{n}\right)\right|^{s}\right)^{1 / 2-1 / p}\left\|y^{*}\right\| .
\end{aligned}
$$

Hence $\|T\| \leq C_{p}\left(\sum_{n \in \sigma}\left|x^{*}\left(e_{n}\right)\right|^{s}\right)^{1 / 2-1 / p}$. Since $X^{*}$ is a GT space, we have

$$
\begin{aligned}
\sum_{n \in \sigma}\left|x^{*}\left(e_{n}\right)\right|^{s} & =\sum_{n \in \sigma}\left|x^{*}\left(e_{n}\right)\right|\left\|T e_{n}^{*}\right\| \\
& \leq B\|T\| \sup _{\varepsilon_{n}= \pm 1}\left\|\sum_{n \in \sigma} \varepsilon_{n} x^{*}\left(e_{n}\right) e_{n}^{*}\right\| \\
& \leq B C_{p}\left(\sum_{n \in \sigma}\left|x^{*}\left(e_{n}\right)\right|^{s}\right)^{1 / 2-1 / p} \sup _{\varepsilon_{n}= \pm 1}\left\|\sum_{n \in \sigma} \varepsilon_{n} x^{*}\left(e_{n}\right) e_{n}^{*}\right\| .
\end{aligned}
$$

Thus,

$$
\left(\sum_{n \in \sigma}\left|x^{*}\left(e_{n}\right)\right|^{s}\right)^{1 / s} \leq B C_{p} \sup _{\varepsilon_{n}= \pm 1}\left\|\sum_{n \in \sigma} \varepsilon_{n} x^{*}\left(e_{n}\right) e_{n}^{*}\right\| .
$$

Since $|\sigma|=N$, Remark 8.3 gives

$$
\sup _{\varepsilon_{n}= \pm 1}\left\|\sum_{n \in \sigma} \varepsilon_{n} x^{*}\left(e_{n}\right) e_{n}^{*}\right\| \leq C^{\prime}\left(\log _{2} N\right) \theta(1 / N)\left\|x^{*}\right\|=C^{\prime}\left(\log _{2} N\right) \theta(1 / N),
$$

where $C^{\prime}$ is independent of $N$. Hence

$$
\left(\sum_{n \in \sigma}\left|x^{*}\left(e_{n}\right)\right|^{s}\right)^{1 / s} \leq B C^{\prime} C_{p}\left(\log _{2} N\right) \theta(1 / N) .
$$

Thus,

$$
\begin{aligned}
\left\|\sum_{n \in \sigma} \eta_{n} e_{n}\right\| & =\sum_{n \in \sigma} \eta_{n} x^{*}\left(e_{n}\right) \leq\left(\sum_{n \in \sigma}\left|x^{*}\left(e_{n}\right)\right|^{s}\right)^{1 / s} N^{1-1 / s} \\
& \leq B C^{\prime} C_{p}\left(\log _{2} N\right) \theta(1 / N) N^{1-1 / s} .
\end{aligned}
$$

Now suppose that $y^{*} \in X^{*}$ with $\left\|y^{*}\right\|=1$. For $a>0$, let

$$
\sigma(a)=\left\{n:\left|y^{*}\left(e_{n}\right)\right| \geq a\right\} \quad \text { and } \quad N(a)=|\sigma(a)| .
$$


Then, for some choice of signs $\left(\eta_{n}\right)$, we have

$$
\begin{aligned}
a N(a) & \leq y^{*}\left(\sum_{n \in \sigma(a)} \eta_{n} e_{n}\right) \leq\left\|\sum_{n \in \sigma(a)} \eta_{n} e_{n}\right\| \\
& \leq B C^{\prime} C_{p}\left(\log _{2} N(a)\right) \theta(1 / N(a)) N(a)^{1-1 / s} \\
& \leq B C C^{\prime} C_{p}\left(\log _{2} N(a)\right) N(a)^{1-1 / s+\varepsilon},
\end{aligned}
$$

using the hypothesis that $\theta(a) \leq C a^{-\varepsilon}$. Thus, for some constant $C^{\prime \prime}$, we have $N(a) \leq C^{\prime \prime} a^{-t}$ provided $t$ satisfies

$$
\frac{1}{r}<\frac{1}{t}<\frac{1}{s}-\varepsilon .
$$

This implies, by a standard calculation, that $\left(e_{n}\right)$ satisfies $M_{r}$.

THEOREM 8.5. Let $\left(e_{n}\right)$ be a semi-normalized thresholding-bounded basis for $X$ satisfying $\theta(a) \leq C a^{-\varepsilon}$, where $0<\varepsilon<1 / 2$. If $X^{*}$ is a GT space, then $\left(e_{n}\right)$ is equivalent to the unit vector basis of $c_{0}$.

Proof. For simplicity, we assume that the basis is normalized (the seminormalized case is similar). Note that $\left(e_{n}\right)$ satisfies $M_{\infty}$. By applying Lemma 8.4 a total of $\left[(1-2 \varepsilon)^{-1}\right]+1$ times, starting with $p=\infty$, we finally deduce that $\left(e_{n}\right)$ satisfies $M_{2}$. Now apply Proposition 8.1.

Corollary 8.6. $c_{0}$ is the unique infinite-dimensional $\mathcal{L}_{\infty}$ space, up to isomorphism, with a quasi-greedy basis. Moreover, $c_{0}$ has a unique quasigreedy basis up to equivalence.

REMARK 8.7. Szarek [20] proved that every Schauder basis of an infinitedimensional $\mathcal{L}_{\infty}$ space contains a subsequence equivalent to the unit vector basis of $c_{0}$. Thus, $c_{0}$ is the only infinite-dimensional $\mathcal{L}_{\infty}$ space, up to isomorphism, with a superdemocratic or a semi-greedy (by Proposition 3.3) basis.

COROLlary 8.8. The disc algebra (regarded as a real Banach space) does not have a quasi-greedy basis.

Corollary 8.9. Let $X$ be an infinite-dimensional Banach space. Then $X$ has a unique normalized (or unique semi-normalized) quasi-greedy basis up to equivalence if and only if $X$ is isomorphic to $c_{0}$.

Proof. The fact that $c_{0}$ has a unique quasi-greedy basis is the second assertion of Corollary 8.6. Suppose that $X$ is a Banach space with a unique (up to equivalence) normalized (respectively, semi-normalized) quasi-greedy basis $\left(e_{n}\right)$. Let $\left(\varepsilon_{n}\right)$ be any choice of signs. Then $\left(\varepsilon_{n} e_{n}\right)$ is also quasi-greedy and normalized (respectively, semi-normalized). So, by uniqueness, $\left(\varepsilon_{n} e_{n}\right)$ is equivalent to $\left(e_{n}\right)$, and so $\left(e_{n}\right)$ is unconditional. In particular, $X$ has a unique unconditional basis up to equivalence. It follows from the LindenstraussZippin theorem [14] that $X$ is isomorphic to $c_{0}, \ell_{1}$ or $\ell_{2}$. But $\ell_{1}$ (cf. [4]) 
and $\ell_{2}$ (cf. [23]) have conditional quasi-greedy bases. Thus $X$ is isomorphic to $c_{0}$.

Acknowledgements. The authors thank Ted Odell for helpful discussions and several useful references.

\section{References}

[1] B. Beauzamy et J.-T. Lapresté, Modèles étalés des espaces de Banach, Travaux en cours, Hermann, Paris, 1984.

[2] J. Bourgain, New Banach space properties of the disc algebra and $H^{\infty}$, Acta Math. 152 (1984), 1-48.

[3] S. J. Dilworth, N. J. Kalton, D. Kutzarova and V. N. Temlyakov, The thresholding greedy algorithm, greedy bases, and duality, Constr. Approx., in press.

[4] S. J. Dilworth and D. Mitra, A conditional quasi-greedy basis of $l_{1}$, Studia Math. 144 (2001), 95-100.

[5] J. Elton, Weakly null normalized sequences in Banach spaces, Ph.D. thesis, Yale Univ., 1978.

[6] R. Gribonval and M. Nielsen, Some remarks on non-linear approximation with Schauder bases, East J. Approx. 7 (2001), 267-285.

[7] A. Grothendieck, Résumé de la théorie métrique des produits tensoriels topologiques, Bol. Soc. Mat. São Paulo 8 (1953), 1-79.

[8] R. C. James, A non-reflexive Banach space isometric with its second conjugate space, Proc. Nat. Acad. Sci. U.S.A. 37 (1951), 174-177.

[9] M. Junge, N. Nielsen, Z. J. Ruan and Q. H. Xu, $\mathcal{C O P} p$ spaces-the local structure of non-commutative $L_{p}$ spaces, preprint.

[10] M. Junge and Z. J. Ruan, Approximation properties for non-commutative $L_{p}$ spaces associated with discrete groups, preprint.

[11] R. A. Komorowski and N. Tomczak-Jaegermann, Subspaces of $\ell_{2}(X)$ and $\operatorname{Rad}(X)$ without local unconditional structure, Studia Math. 149 (2002), 1-21.

[12] S. V. Konyagin and V. N. Temlyakov, A remark on greedy approximation in Banach spaces, East J. Approx. 5 (1999), 365-379.

[13] J. Lindenstrauss and L. Tzafriri, Classical Banach spaces I. Sequence Spaces, Springer, Berlin, 1977.

[14] J. Lindenstrauss and M. Zippin, Banach spaces with a unique unconditional basis, J. Funct. Anal. 3 (1969), 115-125.

[15] B. Maurey and H. Rosenthal, Normalized weakly null sequences with no unconditional subsequence, Studia Math. 61 (1977), 77-98.

[16] E. Odell, Applications of Ramsey theorems to Banach space theory, in: Notes in Banach Spaces, H. E. Lacey (ed.), Univ. of Texas Press, Austin, TX, 1980, 379-404.

[17] A. Pełczyński and I. Singer, On non-equivalent bases and conditional bases in Banach spaces, Studia Math. 25 (1964), 5-25.

[18] G. Pisier, Factorization of Linear Operators and Geometry of Banach Spaces, CBMS Regional Conf. Ser. in Math. 60, Amer. Math. Soc, Providence, RI, 1986.

[19] H. P. Rosenthal, A characterization of Banach spaces containing $l^{1}$, Proc. Nat. Acad. Sci. U.S.A. 71 (1974), 2411-2413.

[20] S. J. Szarek, Bases and biorthogonal systems in the spaces $C$ and $L^{1}$, Ark. Mat. 17 (1979), 255-271. 
[21] B. S. Tsirelson, Not every Banach space contains $\ell_{p}$ or $c_{0}$, Funct. Anal. Appl. 8 (1974), 138-141.

[22] P. Wojtaszczyk, Existence of some special bases in Banach spaces, Studia Math. 67 (1973), 83-93.

[23] —, Greedy algorithm for general biorthogonal systems, J. Approx. Theory 107 (2000), 293-314.

Department of Mathematics

University of South Carolina

Columbia, SC 29208, U.S.A.

E-mail: dilworth@math.sc.edu

Department of Mathematics

University of Missouri

Columbia, MO 65211, U.S.A.

E-mail: nigel@math.missouri.edu
Institute of Mathematics Bulgarian Academy of Sciences Sofia, Bulgaria

Current address:

Department of Mathematics University of Illinois at Urbana-Champaign Urbana, IL 61801, U.S.A. E-mail:denka@math.uiuc.edu

Received July 29, 2002

Final version February 11, 2003 